\title{
Aging-dependent downregulation of SUV39H1 histone methyltransferase increases susceptibility to stress-induced depressive behavior
}

\author{
Jung-Eun Lee
}

Ewha Womans University

So-Young Park

Ewha Womans University

Pyung-Lim Han ( $\sim$ plhan@ewha.ac.kr)

Ewha Womans University https://orcid.org/0000-0002-1735-6746

\section{Research Article}

Keywords: Aging, SUV39H1, Mkp-1, p-CREB, BDNF.

Posted Date: May 27th, 2021

DOl: https://doi.org/10.21203/rs.3.rs-526053/v1

License: (1) This work is licensed under a Creative Commons Attribution 4.0 International License.

Read Full License

Version of Record: A version of this preprint was published at Molecular Neurobiology on September 18th, 2021. See the published version at https://doi.org/10.1007/s12035-021-02529-0. 


\section{Abstract}

Aging induces cellular and molecular changes including gene expression alteration in the brain, which might be associated with aging-dependent increase in vulnerability to stress-induced depression. However, the underlying mechanism is not clearly understood. In the present study, we investigate how aging changes the ability to cope with stress and increases sensitivity to stress. Aged mice have decreased expression of SUV39H1 histone methyltransferase and increased expression of Mkp-1 in the hippocampus. The siRNA-mediated knockdown of SUV39H1 increases Mkp-1 expression and suppresses p-CREB and Bdnf expression in HT22 cells and in the hippocampus of mice. Chromatin immunoprecipitation assays indicate that the levels of $\mathrm{SUV} 39 \mathrm{H} 1$ and methylated histone-H3 bound to the promoter of the $M k p-1$ in the hippocampus are reduced in aged mice. Aged mice exhibit depression-like behavior following weak stress that does not induce depressive behavior in young mice. Rosmarinic acid, a phenolic compound that increases SUV39H1 expression, reverses stress-induced changes of SUV39H1, Mkp-1, and Bdnf expression in the hippocampus via an overlapping but distinct mechanism from those of fluoxetine and imipramine and produces anti-depressive effects. These results suggest that aging increases susceptibility to stress via downregulation of SUV39H1 and changes in SUV39H1-regulated signaling pathways in the hippocampus.

\section{Introduction}

Stress responses proceed with a neuroendocrine reaction, resulting in the release of glucocorticoids (GC) into the blood. GC release in a homeostatic range is vital to activate global metabolic activities of the body and helps cope with intrinsic or extrinsic adverse stimuli $[1,2,3]$. However, when the stress response is activated excessively, it may cause maladaptive changes including structural and functional changes in various brain regions such as the hippocampus, prefrontal cortex, and amygdala $[4,5,6,7]$.

Elderly people have increased GC levels in blood [8, 9], and show blunted responses to stress [10, 11]. Brain aging advances with cellular and molecular changes including accumulation of oxidative stress, increased mitochondrial dysfunction, reduced expression of neurotrophic factors, and impaired cellular stress responses $[9,12]$. These aging-related changes in the brain are likely to be associated with GCinduced events. However, the detailed mechanisms by which aging and stress-induced changes accumulate maladaptive changes in the brain and promote depressive behaviors are not clearly understood.

Mitogen-activated protein kinase phosphatase-1 (Mkp-1) plays an important role in stress-induced adaptive changes in the brain. Mkp-1 is rapidly upregulated by stress in the hippocampus $[13,14]$. While activated ERK phosphorylates CREB, which in turn regulates the expression of neurotrophic factors including BDNF and NPY [16, 17], increased Mkp-1 decreases the activity of ERKs, p38 kinases and JNKs [15]. p-CREB and BDNF are reduced in the hippocampus of aged animals [18-20]. The Mkp-1 gene promoter regions contain potential glucocorticoid receptor (GR) binding sites between - 1421 and - 1118, and between -717 and -702 [21, 22]. GC treatment in HT22 cells increases Mkp-1 expression and GR 
binding to its proximal promoter [22]. Aged mice show increased expression of Mkp-1 in the hippocampus [23]. These results suggest that aging-induced increased expression of Mkp-1 could decrease $\mathrm{p}$-CREB and BDNF expression in the hippocampus and stress-induced behavioral deficits. However, the underlying mechanism needs to be elaborated.

Epigenetic factors are critical players in the sustained maintenance of depressive behaviors induced by chronic stress $[24,25]$. The histone methyltransferase SUV39H1, which adds tri- or dimethyl groups to histone-3 lysine 9 (H3K9), is downregulated in the hippocampus of mice treated with chronic stress, and reduced expression of SUV39H1 in the hippocampus causes depressive behaviors [22]. Furthermore, SUV39H1 expression is reduced in aged brains [23]. Therefore, questions are raised about whether agingdependent change in SUV39H1 upregulates Mkp-1 and facilitates stress-induced depressive behaviors and if a strategy targeting SUV39H1 or Mkp-1 is beneficial for the recovery of stress-dependent changes in aged brains.

In the present study, we demonstrate that GC and aging-dependent downregulation of SUV39H1 increases Mkp-1 expression and reduces Bdnf expression in the hippocampus and increases the risk for stress-induced depressive behaviors in aged mice.

\section{Materials And Methods}

\section{Materials}

Corticosterone, RU486, imipramine, and fluoxetine were purchased from Sigma-Aldrich (St. Louis, MO, USA). Rosmarinic acid was purchased from Tocris (Bristol, UK). They were dissolved in $0.9 \%$ saline.

\section{Methods}

\section{Animals}

Seven-week-old male C57BL/ 6 mice were purchased from Daehan BioLink Inc. (Eumsung, Chungbuk, Korea). Aged C57BL/6J mice were obtained from the Jackson Laboratory (Bar Harbor, ME, USA) and were raised in the laboratory. Mice were housed in pairs in a standard clear plastic cage filled with chopped wood particles (TAPVEI, Paekna, Estonia). They had free access to food and water in a temperature $\left(23^{\circ} \mathrm{C}\right)$ - and humidity (50-60\%)-controlled environment under 12-h light/dark cycle conditions (from 7:00 AM to 7:00 PM). All animals were handled in accordance with the animal care guidelines of Ewha Womans University (IACUC 16-018).

\section{Restraint stress}

Mice were subjected to restraint stress as described previously [26, 27]. Mice were randomly assigned into experimental groups and housed in pairs in a plastic cage 5 days before placing in the restraint regimen. Mice were individually placed head first into a well-ventilated 50-ml polypropylene conical tube with holes for ventilation and restrained against back-and-forth movement. This treatment was applied for 2-h per 
day and was repeated for 14 days or the number of days indicated. After each daily session of restraint, the mice were returned to their home cages.

\section{Drug administration}

Mice were intraperitoneally injected with rosmarinic acid (30 mg/ $\mathrm{Kg})$, fluoxetine $(20 \mathrm{mg} / \mathrm{Kg})$, or imipramine $(20 \mathrm{mg} / \mathrm{Kg}) 30 \mathrm{~min}$ before the start of each 2-h restraint stress in the co-treatment paradigm. Cell culture and drug treatment

HT22 mouse hippocampal cells were cultured as described previously [22, 23]. HT22 cells were cultured in DMEM (LM-001-05; Welgene, Gyeongsan, Korea) supplemented with 10\% heat-inactivated fetal bovine serum (FB02-500; Serum Source, NC, USA) and antibiotics (penicillin and streptomycin) (LS-202-02; Welgene) at $37^{\circ} \mathrm{C}$ in a humidified incubator gassed with $95 \%$ air and $5 \% \mathrm{CO}_{2}$.

Cells grown to $70-80 \%$ confluence were treated with corticosterone, RU486, or drugs in DMEM containing $1 \%$ fetal bovine serum. After $24 \mathrm{~h}$, cells were washed with phosphate-buffered saline (PBS) and harvested for experiments. Corticosterone, RU486, imipramine, fluoxetine and rosmarinic acid were treated at the dose indicated.

HT22 cells were transfected with siRNA using Lipofectamine reagent (Invitrogen, Carlsbad, CA, USA) as described previously [22]. siRNA transfection was carried out in DMEM containing $1 \%$ FBS. The final concentration of siRNA was $100 \mathrm{nM}$, and the concentration of Lipofectamine-2000 was $7.5 \mu \mathrm{l} /$ well.

The siRNAs used were siRNA-control (SN-1012), siRNA-Mkp-1 (\#1351659, NM_013642.1), siRNA-Creb (\#1342686, NM_009952.2), and siRNA-SUV39H1 (\#1433203, NM_011514.1), which were obtained from Bioneer Co. (Daejeon, Korea).

\section{Measurement of corticosterone levels}

Corticosterone levels were measured using an ELISA kit (ADI-901-097; Enzo Life Science, Farmingdale, NY, USA) as described previously [23]. Briefly, immediately after the 2-h restraint, mice were anesthetized with $2.5 \%$ avertin (intraperitoneal injection) at a dose of $20 \mu \mathrm{g} / \mathrm{g}$ body weight. Blood was collected from the heart between 9:00 AM to 12:00 PM. Blood samples were centrifuged at 3,000 rpm at $4^{\circ} \mathrm{C}$ for $15 \mathrm{~min}$ in a microcentrifuge, and the supernatant was collected. The supernatant $(100 \mu \mathrm{L})$ of each sample was diluted with the steroid displacement reagent solution of the ELISA kit. They were then dispensed into new wells of the ELISA kit plate and incubated with antibody reagent on a plate shaker. After a 2-h reaction, each well was washed 3 times with wash solution from the ELISA kit. Then, p-nitrophenyl phosphatate solution was added and incubated for $1 \mathrm{~h}$. The reaction was stopped with stop solution, and the plate was read immediately at $405 \mathrm{~nm}$.

\section{Immunohistochemistry}

Immunohistochemical analyses were carried out as described previously [22]. Briefly, mice were perfused with $4 \%$ paraformaldehyde by a trans-cardiac method, and the brains were surgically removed and immersed in the same fixative overnight at $4^{\circ} \mathrm{C}$. Brains were coronally cut into $40-\mu \mathrm{m}$ thick sections using 
a vibratome (Leica VT 1000S; Leica Instruments, Nussloch, Germany). Brain sections were incubated at RT for $1 \mathrm{~h}$ with $5 \%$ BSA using a free-floating method and were reacted with primary antibody in $5 \%$ BSA overnight at $4^{\circ} \mathrm{C}$. After washing 3 times with $1 \times$ PBST, the sections were incubated with a secondary antibody for $1 \mathrm{~h}$. After washing with $1 \mathrm{x}$ PBST, stained sections were mounted on a gelatin-coated slide with a DAPI staining mounting solution ( $\mathrm{H}-1200 ;$ Vector Lab.). The stained sections were analyzed using an Olympus BX 51 microscope equipped with a DP71 camera and MetaMorph Microscopy Automation \& Image Analysis software (Molecular Devices, San Jose, CA, USA).

The primary antibodies used were p-CREB (06-519, Millipore, Billerica, MA, USA; 1:500; RRID:AB_310153), p-ERK (4370s, Cell Signaling, Danvers, MA, USA; 1:500; RRID:AB_2315112), MKP-1 (sc-271684, Santa Cruz, TX, USA; 1:200; RRID:AB_10708413), MAP2 (05-346; Millipore, 1:1000; RRID: AB_309685), Doublecortin (sc-8066, Santa Cruz; 1:1000; RRID:AB_2088494), and Ki67 (VP-K451, Vector Lab.; 1:1000; RRID:AB_2314701). The secondary antibodies used were anti-mouse IgG tagged with Dylight 488 (DI2488, Vector Lab., Burlingame, CA, USA) or rabbit IgG conjugated Dylight 594 (DI-1094, Vector Lab.).

\section{Western blot analyses}

Western blot analysis was performed as described previously [30]. Hippocampal tissue or harvested cells were homogenized in homogenization buffer $(50 \mathrm{mM}$ Tris-HCl, pH 8.0, $150 \mathrm{mM} \mathrm{NaCl}, 1 \%$ Nonidet P-40, $0.1 \%$ SDS, and $0.1 \%$ sodium deoxycholate) containing a cocktail of protease inhibitors (Roche, Mannheim, Germany) on ice using an Epishear probe sonicator (Active Motif, Carlsbad, CA, USA) at a 40\% power outlet with two rounds of 10 -seccond pulses. The homogenate was centrifuged at $13,000 \mathrm{~g}$ at $4^{\circ} \mathrm{C}$ for $10 \mathrm{~min}$, and the supernatant was collected. Protein concentration was measured by the Bradford method (Bio-Rad Laboratories. Hercules, CA, USA). Tissue or cell proteins $(20 \mu \mathrm{g})$ were mixed with $6 \mathrm{x}$ sample loading buffer, and boiled for $5 \mathrm{~min}$. The samples were resolved on SDS-PAGE, and then they were transferred onto a PVDF membrane (Bio-Rad Laboratories). Blots were incubated with blocking solution containing 1\% BSA in TBST (150 mM NaCl, 50 mM TRIS-Cl buffer, $\mathrm{pH} \mathrm{7.4,} \mathrm{0.1 \%} \mathrm{Tween} \mathrm{20)} \mathrm{for} 1$ $\mathrm{h}$, followed by incubation with a primary antibody in blocking solution for $3 \mathrm{~h}$ at RT. The blots were washed 3 times with TBST and then incubated with a secondary antibody of anti-mouse IgG-HRP (GTX213111-01; 1:1,000, GeneTex, Irvine, CA, USA) dissolved in TBST at RT for $1 \mathrm{~h}$. Immunoblots were visualized using a PicoEPD Western Reagent Kit (EBP-1073; ELPis Biotech, Daejeon, Korea). Western blot images were quantified using Image-ProPremier 6.0 (MediaCybernetics, MD, USA).

The primary antibodies used were anti-p-CREB (06-519, Millipore; 1:1000; RRID:AB_310153), anti-CREB (sc-186, Santa Cruz; 1:1000; RRID:AB_2086021), and anti-ß-actin (sc-47778; 1:1,000, Santa Cruz). The secondary antibodies used are goat anti-mouse IgG (GTX213111, GeneTex, Irvine, CA, USA) and goat antirabbit lgG (GTX213110, GeneTex).

\section{Quantitative real-time PCR}

Quantitative real-time PCR was carried out as described previously [22, 23]. Briefly, total RNA from the hippocampal tissues and cultured cells was isolated with TRIzol reagent (15596-018; Invitrogen). The concentration of isolated RNA was measured with NanoDrop (Thermo Fisher Scientific, Waltham, MA, 
USA). The RNA was treated with DNase I (Promega, Madison, WI, USA) to prevent genomic DNA contamination, and then $1 \mu \mathrm{g}$ of total RNA was converted to cDNA using a reverse transcriptase system (LeGene Bioscience \#6100; San Diego, CA, USA). Real-time PCR was performed with $4 \mu$ l of cDNA (1/8 dilution of the converted cDNA), $10 \mu$ of $2 x$ iQ ${ }^{\text {TM }}$ SYBR ${ }^{\circledR}$ Green Supermix (Bio-Rad Laboratories), and $1 \mu \mathrm{l}$ each of $5 \mathrm{pmol} / \mu \mathrm{l}$ forward and reverse primers in a volume of $20 \mu \mathrm{l}$ using the CFX 96 Real-Time PCR System Detector (Bio-Rad Laboratories).

Real-time PCR was carried out using the following primers: 5'-GCGCTCCACTCAAGTCTTCT-3' and 5'AGAGGGGTACTACAGGAGCT-3' for Mkp-1, 5'-TGGCTGACACTTTTGAGCAC-3' and 5'GTTTGCGGCATCCAGGTAAT-3' for Bdnf, 5'-GAGGCAGCAAGAGAATGTCG-3' and 5'CCAGTCCATTCTCCACCGTA-3' for Creb, and 5'-CAACCTTGATGAGCGACTAC-3' and 5'CCATTCGGGTACTCTCCA-3' for SUV39H1, 5'-AGAAGGTGGTGA AGCAGGCATC-3' and 5'CGAAGGTGGAAGA GTGGGAGTTG-3' for Gapdh, and 5'-GCTGCCATCTGTTTTACGG-3' and 5'TGACTGGTGCCTGATGAACT-3' for L32.

\section{Chromatin immunoprecipitation (ChIP)-qPCR analyses}

ChIP-qPCR assays were carried out as described previously $[22,23]$ using a ChIP-IT® Express Kit (102026; Active Motif). Hippocampal tissue (40-50 mg) obtained from 6 mice was pooled and minced with a razor blade. Samples were then incubated in $7 \mathrm{ml}$ of $1 \%$ formaldehyde (F1635; Sigma-Aldrich) in 1x PBS for 8 min to cross-link the proteins and DNA. Cross-linking reaction between proteins and DNA was stopped by adding $5 \mathrm{ml}$ of $0.125 \mathrm{M}$ glycine and incubating for $5 \mathrm{~min}$. They were then homogenized using a Dounce homogenizer (357542; Wheaton, Millville, NJ, USA), and the homogenate was centrifuged at $1,250 \mathrm{~g}$ for $5 \mathrm{~min}$. The pellet was collected and resuspended in $500 \mu \mathrm{l}$ of lysis buffer containing proteinase cocktail $(0.5 \mathrm{x}$, final) and PMSF $(0.5 \mathrm{mM}$, final), and incubated on ice for $30 \mathrm{~min}$. The lysates then were homogenized again and washed with $1 x$ PBS twice. The pellet containing fixed chromatin was resuspended in $350 \mu \mathrm{l}$ of shearing buffer containing proteinase inhibitors and PMSF. They were then sheared into 200-800 bp fragments by sonication on ice using an Epishear probe sonicator (Active Motif) with 20-second pulses at 50-second intervals at 35\% power, repeated 20 times. The sheared chromatin was centrifuged at 22,000 $\mathrm{g}$ for $10 \mathrm{~min}$, and the supernatant was saved for immunoprecipitation. The amount of DNA in the sheared chromatin samples was quantified using NanoDrop (Thermo Fisher Scientific). Successful chromatin shearing was confirmed by agarose gel electrophoresis. Sheared chromatin samples were stored at $-80^{\circ} \mathrm{C}$ until use.

For the immunoprecipitation (IP) reaction, $10 \mu \mathrm{g}$ of sheared chromatin of each sample was mixed with 2 $\mu \mathrm{g}$ of primary antibody, $20 \mu \mathrm{l}$ of Protein G magnetic beads, $10 \mu$ of $10 x$ ChIP buffer 1 from the ChIP Assay Kit (Active Motif), and $1 \mu \mathrm{l}$ of proteinase inhibitor cocktail in a volume of $100 \mu \mathrm{l}$, and was incubated at $4^{\circ} \mathrm{C}$ overnight. The primary antibodies used were SUV39H1 (05-615, Millipore; RRID:AB_2196724), dimeH3K9 (ab1220, Abcam, Cambridge, UK; RRID:AB_449854), or trimeH3K9 (ab8898, Abcam; RRID:AB_306848). 
The reacted IP products were washed with ChIP buffer I, followed by ChIP buffer II twice using a magnetic rack (Active Motif). After the final wash, the beads were eluted with $50 \mu$ l of elution buffer and incubated at RT for 15 min with intermittent agitation. Then, each sample was added to $50 \mu$ of reverse crosslinking buffer, and the supernatant containing eluted chromatin was carefully taken on a magnetic rack. The input DNA (10 $\mu \mathrm{l})$ was also prepared as the mixture with $88 \mu \mathrm{l}$ of ChIP buffer II and $2 \mu \mathrm{l}$ of $5 \mathrm{M} \mathrm{NaCl}$. After incubation at $95^{\circ} \mathrm{C}$ for $15 \mathrm{~min}, 1 \mu \mathrm{g}$ of Proteinase $\mathrm{K}$ was added to the reaction mixture and incubated at $37^{\circ} \mathrm{C}$ for $1 \mathrm{~h}$. Followed by the addition of $2 \mu$ l of proteinase $\mathrm{K}$ stop solution, the resulting reaction was regarded as immunoprecipitated DNA and used for real-time PCR.

The real-time PCR reaction was carried out with $4 \mu$ of the immunoprecipitated DNA, $10 \mu$ of $2 x \mathrm{iQ}^{\mathrm{TM}}$ SYBR® Green Supermix (Bio-Rad Laboratories), and $1 \mu \mathrm{l}$ each of $5 \mathrm{pmol} / \mu \mathrm{l}$ forward and reverse primers in a volume of $20 \mu \mathrm{l}$ using the CFX 96 Real-Time PCR System Detector (Bio-Rad Laboratories). One-tenth of the input DNA was used for quantitative PCR to control the relative amounts of DNA fragments in immunoprecipitation and normalized for quantification. Real-time PCR was carried out using the following primers: 5'-CCAGAGGACAGGAGGGTG-3' and 5'-TTGAATGGCAGACACGTGAC-3' for Mkp-1 P1, for Mkp-1 P1, and 5'-AGTGGAGATGAAAGGCACGA-3' and 5'-GACTTGGGTAGAGCTCCACA-3' for Mkp-1 P2.

\section{Stereotaxic injections of siRNA}

Stereotaxic injection of siRNA was carried out as described previously [22]. Briefly, mice were anesthetized with a $3.5: 1$ mixture of ketamine hydrochloride $(50 \mathrm{mg} / \mathrm{ml})$ and xylazine hydrochloride $(23.3 \mathrm{mg} / \mathrm{ml})$ at a dose of $2.5 \mu \mathrm{l} / \mathrm{g}$ body weight. Each siRNA $(50 \mathrm{ng} / \mu \mathrm{l})$ was mixed with 2.5 volume of Neurofect transfection reagent (T800075; Genlantis, San Diego, CA, USA) and 0.5 volume of $50 \%$ sucrose, and the mixture was incubated for $20 \mathrm{~min}$. A $1.8 \mu \mathrm{l}$ volume of the siRNA mix $(7.5 \mathrm{ng} / \mu \mathrm{l})$ was injected into each CA3 region (stereotaxic coordinate: $A P,-1.9 ; \mathrm{ML}, \pm 2.1 ; \mathrm{DV},-2.1 \mathrm{~mm}$ ) at $0.2 \mu \mathrm{l} / \mathrm{min}$ using a stereotaxic injection system (Vernier Stereotaxic Instrument, Leica Biosystems, Wetzlar, Germany) and a Hamilton syringe with a 30-G needle. The animals were sacrificed to analyze the gene expression at $72 \mathrm{~h}$ after siRNA injection.

siRNA-control (SN-1012), siRNA-Mkp-1 (\#1351659, NM_013642.1), and siRNA-SUV39H1 (\#1433203, NM_011514.1) were purchased from the Bioneer Co (Daejeon, Republic of Korea).

\section{Behavioral analyses}

Behavioral tests were performed during the light cycle (9 A.M. 4 P.M) as described previously [22, 26]. Mice were brought to the behavior testing room $30 \mathrm{~min}$ before the start of each behavioral test. Background sound in the testing room was masked with $65 \mathrm{~dB}$ of white noise by a white noise generator (HDT Korea, Seoul, Korea). The light was lit with indirect illumination by 20 lux for the U-shaped twochoice field and 250 lux for the tail suspension test (TST) and forced swimming test (FST). Animal behaviors in the tests were recorded using a computerized video tracking system (SMART, PanLab, Spain) or a webcam recording system (HD Webcam C210, Logitech, USA).

Social interaction time and distance in the U-shaped two-choice field were measured using an equipped program (SMART, PanLab). Immobility time in the tail suspension test and forced swimming test was 
recorded in a blind manner. All behavioral tests were conducted in a randomized fashion and/or in an alternative manner depending on test order and position in the U-shaped two choice field (e.g., left vs. right).

\section{Sociability test}

The sociability test using a U-shaped two-choice field was performed as described previously [22, 26]. The U-shaped two-choice field consisted of symmetrical two-spaces with a partitioning wall $(40 \mathrm{~cm})$ in the open field $(45 \times 45 \mathrm{~cm})$. Each symmetrical space had both closed and open square zones, forming a " $U$ shaped two-choice field" [26]. Each side of the closed square contained a circular grid cage $(12 \mathrm{~cm}$ in diameter $\times 33 \mathrm{~cm}$ in height) with either a social target or an empty cage. The space containing a social target was defined as the target field, and the other side with an empty cage was defined as the nontarget field. Subject mice were individually introduced in the middle of the U-shape field and allowed to freely explore $5 \mathrm{~min}$ before the test. After habituation, the mice were returned to their home cage for $1 \mathrm{~min}$ and then exposed again for 10 min to the U-shaped field with a social target in a grid cage on one side and an empty cage on the other. Normal B6 male mice were used as a social target, and each target mouse served 3-4 times. All animals, including social targets, were housed in pairs in standard plastic home cages.

\section{Tail suspension test}

The tail suspension test (TST) was performed as described previously [22]. Mice were individually suspended by fixing their tails to the ceiling of a white wooden box using adhesive tape, thereby being held $50 \mathrm{~cm}$ above the surface of a table. During a 6 min period, the cumulative immobility time of each mouse was counted.

\section{Forced swimming test}

The forced swimming test (FST) was carried out as described previously [22]. Mice were placed individually in a Plexiglas cylinder $\left(15 \mathrm{~cm}\right.$ in diameter $x 27 \mathrm{~cm}$ in height) filled with water at $24^{\circ} \mathrm{C}$ at a depth of $14 \mathrm{~cm}$. During a 6 min period, mice were put in the Plexiglas cylinder and their escape-related mobility behavior was measured during the last $5 \mathrm{~min}$. Immobility time was defined as the sum of time during the floating behavior in which all limbs were motionless.

\section{K-means clustering}

K-means cluster analyses were carried out using SPSS statistics 25 software (IBM SPP Statistics, NY, USA) as described previously [23]. K-means clustering is an unsupervised machine-learning algorithm that classifies data points into similar groups. Depending on the $k$ values, all individual points are assigned to the nearest cluster.

\section{Statistical analyses}

GraphPad PRISM 6.0 software (GraphPad Software. Inc., CA, USA) was used for statistical analyses. Two-sample comparisons were carried out using the Student's t-test, and multiple comparisons were made using a one-way ANOVA followed by the Newman-Keuls post hoc test or two-way ANOVA followed 
by the Bonferroni post hoc test. All data are presented as mean \pm SEM, and statistical significance was accepted at the $5 \%$ level.

\section{Results}

\section{Aging decreased SUV39H1 expression while increasing Mkp-1 expression in the hippocampus.}

Recently we reported that young mice exposed to chronic stress have decreased expression of SUV39H1 in the hippocampus, which causes sustained upregulation of Mkp-1 [22]. In the present study, we continued to investigate whether SUV39H1 and Mkp-1 have a role in the aging brain. The transcript levels of Mkp-1 increased in the hippocampus in an aging-dependent manner, which was in contrast to the aging-dependent decrease of SUV39H1 expression in the hippocampus (Fig. 1a,b). The siRNA-mediated knockdown of SUV39H1 in the hippocampus increased the expression of Mkp-1 (Fig. 1c,d). These results suggest that the aging-dependent reduction in the expression of $\mathrm{SUV} 39 \mathrm{H} 1$ is closely related to the aginginduced increase in the expression of Mkp-1. Chromatin immunoprecipitation (ChIP) analysis indicated that the level of SUV39H1 bound to the promoter region of the $M k p-1$ was significantly reduced in the hippocampus of aged mice (18 months or $18 \mathrm{M}$ ) compared to that of young mice (2 M) (Fig. 1e,f). Consistently, the levels of tri- and di-methylated histone-H3 bound to the promoter region of the $M \mathrm{kp}-1$ were also reduced in aged mice compared to those in young mice (Fig. $1 \mathrm{~g}, \mathrm{~h}$ ). These results suggest that the aging-dependent decrease in the expression of SUV39H1 could increase Mkp-1 expression in the hippocampus.

\section{Reduced expression of SUV39H1 and increased expression of Mkp-1 decreased p-CREB and Bdnf expression.}

Aged mice (18 M) had increased basal corticosterone levels in the blood compared to those of young mice (2 M) (Fig. 2a), which is consistent with the previous report [23]. Aged mice (18 M) had reduced levels of p-CREB and Bdnf expression in the hippocampus (Fig. 2b,c). Therefore, we asked whether the reduced expression of CREB and Bdnf results from GC-induced changes in the expression of SUV39H1 and Mkp-1. GC treatment in HT22 hippocampal cells decreased the levels of p-CREB and Bdnf expression (Fig. 2d,e). Moreover, GC treatment in HT22 cells decreased the expression of SUV39H1 and increased the expression of Mkp-1, in which both GC-induced changes were blocked by co-treatment with RU486, a GR antagonist (Fig. 2g,h). Consistently, siRNA-mediated knockdown of SUV39H1 in HT22 cells increased the expression of Mkp-1 and decreased the levels of p-CREB and Bdnf expression (Fig. 2h-j). In contrast, siRNA-mediated knockdown of Mkp-1 in HT22 cells increased the levels of p-CREB and Bdnf expression (Fig. 2k-m). The siRNA-mediated knockdown of CREB in HT22 cells decreased Bdnf expression (Fig. 2n,o). Together, these results suggest that GC-induced downregulation of SUV39H1 in HT22 cells induces Mkp-1 expression, which in turn negatively regulates p-CREB and Bdnf expression (Fig. 2p).

Next, we examined if Mkp-1 could regulate p-CREB and Bdnf expression in the hippocampus of mice. Immunohistochemical analysis indicated that Mkp-1 and p-CREB were co-localized at the single-cell level in CA3 pyramidal neurons of the hippocampus, and their expression levels were negatively correlated 
(Fig. 3a,b). Chronic stress decreases p-ERK1/2 and p-CREB, which downregulates BDNF expression [31, 32]. Inhibition of ERK $1 / 2$ by treatment with U0126 (an ERK inhibitor) in HT22 cells reduced the expression of Bdnf (Fig. 3c). The siRNA-mediated knockdown of Mkp-1 in the hippocampus increased Bdnf expression (Fig. 3d,e) and increased the level of p-CREB (Fig. 3f,g). These results suggest that Mkp-1 negatively regulates Bdnf expression via p-ERK and p-CREB (Fig. 3h).

\section{Rosmarinic acid, imipramine, and fluoxetine blocked stress-induced changes of SUV39H1, Mkp-1, and Bdnf expression in an overlapped, but slightly different, way.}

Rosmarinic acid (RA) is a phenolic compound that functions as a transcription inducer of SUV39H1 22]. In HT22 cells, RA treatment (50 and $100 \mu \mathrm{M}$ ) blocked GC-induced reduced expression of SUV39H1 (Fig. 4a), GC-induced increased expression of Mkp-1 (Fig. 4b), and GC-induced reduced expression of Bdnf (Fig. 4c). Imipramine (IMl; 50 and $100 \mu \mathrm{M}$ ) or fluoxetine (FLX; 5 and $10 \mu \mathrm{M}$ ) treatment also reversed the GC-induced downregulation of SUV39H1 and Bdnf expression (Fig. 4a,c), but not the GC-induced increased expression of Mkp-1 (Fig. 4b). We found that high doses of FLX (15 $\mu \mathrm{M}$ and higher) produced cytotoxic effects in HT22 cells, and therefore, $5 \mu \mathrm{M}$ and $10 \mu \mathrm{M}$ of FLX were used in the present study. Overall, these results suggest that RA, IMI, and FLX can reverse GC-induced reduction in the expression of SUV39H1 and Bdnf and that RA can suppress the GC-induced increased expression of Mkp-1. However, IMI and FLX do not block GC-induced increased expression of Mkp-1 in HT22 cells.

Next, we examined whether and how RA, IMI, and FLX treatment modifies stress-induced changes of SUV39H1, Mkp-1, and Bdnf expression in the brain. A single 2-h restraint event decreased the expression of SUV39H1 and Bdnf and increased the expression of Mkp-1 in the hippocampus. The stress-induced gene expression changes were augmented more than those by a single 2-h restraint after treatment with 5 days of 2-h restraint and even further after 14 days of 2-h restraint (Fig. 4e-h), which mirrored the transcriptional changes of the genes after GC-treatment over time in HT22 cells (Fig. 2e-g). These results suggest that the stress-induced decrease in the expression of SUV39H1 and Bdnf and the increased expression of Mkp-1 in the hippocampus might be regulated by the GC-dependent mechanism.

Mice exposed to daily 2-h restraint for 14 days (RST14d) had reduced expression of SUV39H1 and Bdnf and increased expression of Mkp-1 in the hippocampus. In contrast, mice treated with RA each day before the 2-h restraint for 14 days had control levels of SUV39H1 and Bdnf expression, and a control level of Mkp-1 expression (Fig. 4i-I). The antidepressant FLX blocked stress-induced changes of SUV39H1, Bdnf, and Mkp-1 expression in the hippocampus in the same way as RA (Fig. 4i-I). IMI restored the stressinduced decrease in the expression of SUV39H1 and Bdnf, but it did not suppress the stress-induced increase in the expression of Mkp-1 (Fig. 4i-I).

In the behavioral tests, mice treated with RA before the 2-h restraint for 14 days had increased sociability in the social interaction test (SIT), and reduced immobility time in the TST and FST (Fig. 4m-o). Mice treated with IMI before RST14d had increased sociability in the SIT, and reduced immobility time in the TST and FST. Mice treated with FLX before the 2-h restraint for 14 days also had increased sociability in the SIT, and reduced immobility time in the TST, but they had increased immobility in the FST (Fig. 4m-o). 
Immunohistochemical analysis revealed that mice subjected to RST14d had reduced levels of MAP-2 staining, a marker of dendritic processes, in pyramidal neurons of the CA1 and CA3 of the hippocampus, whereas mice that received RA injection before the 2-h restraint for 14 days had enhanced MAP2-stained dendritic morphology of CA1 and CA3 pyramidal neurons (Fig. 5a-d).

Mice subjected to RST14d had a reduced number of new neurons stained by anti-Ki67, a cell proliferation marker (Fig. 5e,f), and a reduced number and reduced levels of dendritic processes of developing neurons stained by anti-doublecortin (DCX), a marker for differentiating neurons (Fig. $5 \mathrm{~g}-\mathrm{i}$ ), in the dentate gyrus of the hippocampus compared to control mice. In contrast, mice that received RA injection before the 2-h restraint for 14 days had an increased number of Ki-stained cells and increased number of DCX-stained neurons in the dentate gyrus relative to RST14d-treated mice. These results suggest that RA protects stress-induced dendritic atrophy of pyramidal neurons in the hippocampus and blocks stress-induced reduction in neurogenesis in the dentate gyrus.

\section{Aged mice were highly sensitive to stress-induced depression, which was moderated by RA.}

Next, we examined whether RA can be used for the treatment of stress-induced depressive behaviors in aged mice. Naïve aged mice (14 M) showed control levels of social interaction in the SIT and control levels of immobility time in the FST, which were comparable to those of naïve young mice (2 M) (Fig. 6ad). Aged mice treated with daily 2-h restraint for 5 days (RST5d), a condition which did not produce depressive-like behavior in young mice, exhibited a slightly reduced sociability, although the difference was not statistically significant, however, immobility time in the FST was significantly increased. In contrast, aged mice treated with RA before the 2-h restraint for 5 days exhibited increased sociability in the SIT and reduced immobility time in the FST (Fig. 6a-d).

Analysis of the behavioral performance of individual animals distributed in the sociability x FST matrix followed by $K$-means clustering, an unsupervised machine-learning algorithm, indicated that the members of the young mouse control, young mice with RST5d, aged mouse control, and the aged mice with RST5d were grouped into two clusters; cluster 1 contained the individuals distributed mostly in the quadrants $1 / 4$ in the sociability x FST matrix, and cluster 2 contained the individuals distributed mostly in the quadrants 2/3 (Fig. 6e). Individuals of the aged mouse group subjected to RST5d were located in cluster 1, which exhibited low sociability and high immobility, while most individuals of the aged mice treated with RA prior to the 2-h restraint for 5 days were in cluster 2, which contained the young mouse control, the young mice with RST5d, and the aged mouse control (Fig. 6e). The \% composition of young control mice and aged control mice in cluster 1 was $57 \%$ for both groups, although the detailed distribution of individuals within the cluster 1 was not overlapped. After RST5d, the \% composition of young mice and aged mice in cluster 1 shifted from $57-43 \%$ and from $57-100 \%$, respectively (that is, $57 \%$ and $100 \%$, respectively, of the group members were positioned in cluster 2 after RST5d) (Fig. 6e,f). More interestingly, when RA was injected before the 2-h restraint for 5 days, the \% composition of young mice with RST5d and aged mice with RST $5 d$ in cluster 1 shifted from $43-100 \%$ and from $100-50 \%$, respectively (that is, $100 \%$ and $50 \%$, respectively, of the group members were positioned in cluster 2 after RA treatment) (Fig. 6e,f). 
The transcript levels of SUV39H1 and Mkp-1 in the hippocampus were decreased and increased, respectively, in aged mice (14 M) compared to those in young mice (2 M) (Fig. 6g,h). Bdnf expression in aged mice was lower than that in young mice (Fig. 6i). After RST5d, the transcript levels of SUV39H1 were reduced and the transcript levels of Mkp-1 were enhanced in both young and aged mice (Fig. $6 \mathrm{~g}, \mathrm{~h}$ ), and the transcript level of Bdnf was reduced in aged mice compared to that in young mice (Fig. 6i). In contrast, RA treatment before the 2-h restraint for 5 days blocked the RST5d-induced reduction in the expression of SUV39H1 and Bdnf and the increase in the expression of Mkp-1 in both young mice and aged mice (Fig. 6g-i).

\section{Discussion}

The present study demonstrates that aged brain had reduced SUV39H1 expression, which resulted in the increased expression of Mkp-1 and reduced expression of p-CREB and Bdnf in the hippocampus (Figs. 1 and 6). Aging-dependent changes in the expression of SUV39H1, Mkp-1, p-CREB and Bdnf mirrored the changes in the expression of those genes in HT22 cells after exposure to GC (Fig. 2) and the changes of the same factors in the hippocampus in mice exposed to chronic stress (Figs. 3 and 4). These results suggest that aging-induced changes of SUV39H1, Mkp-1, p-CREB and Bdnf expression are associated with GC-induced changes. Indeed, aged mice (18 M) had increased basal GC levels (Fig. 2a), and SUV39H1, Mkp-1, p-CREB and Bdnf levels in the hippocampus of aged mice were further changed after exposure to moderate stress (Fig. 6). GC-induced SUV39H1 downregulation and Mkp-1 upregulation could amplify a vicious cycle of SUV39H1-dependent upregulation of Mkp-1 and conversely Mkp-1dependent downregulation of SUV39H1 [22]. Furthermore, SUV39H1 downregulation could change the expression of PP2A, p-AMPK, PPARg and NADPH oxidase, which are also regulated by GC [22, 23]. These results suggest that stress-induced downregulation of SUV39H1 in aged mice might change divergent signaling pathways which result in reduced stress coping ability.

Chronic restraint stress produces depressive-like behaviors, whereas siRNA-mediated knockdown of Mkp1 in the CA3 was sufficient to block stress-induced depressive-like behaviors [22]. Young mice (2 M) that were exposed to RST5d had reduced SUV39H1 expression and increased Mkp-1 expression in the hippocampus. However, they did not exhibit depression-like behaviors (Fig. 6). Aged mice had reduced SUV39H1 expression and increased Mkp-1 expression in the hippocampus but they did not exhibit depression-like behaviors (Fig. 6). These results suggest that RST5d-induced changes of SUV39H1 and Mkp-1 in the hippocampus and aging-induced changes of the same factors are in subthreshold levels. Furthermore, aging-dependent changes advance slowly and progressively with other changes including increased accumulation of oxidative stress, increased mitochondrial dysfunction, and impaired cellular responses to stress $[9,12]$. It could be possible that SUV39H1 and Mkp-1-dependent changes are affected by other aging-dependent accumulated factors in the brain. For example, chronic stress decreases $\mathrm{p}$-ERK and p-CREB levels in a GC- and Mkp-1-dependent manner in the hippocampus [22]. However, p-ERK and pCREB levels can be reduced by oxidative stress and increased levels of cytokines, and those changes could in turn reduce Bdnf expression [33, 34]. Therefore, GC-induced changes of SUV39H1 and Mkp-1 
pathways in aged brains could be amplified by various factors acting on PP2A, p-AMPK, and PPARY pathways in the brain, and the changes might increase susceptibility to stress coping.

Our results suggest RA, IMI, and FLX all offer anti-depressant effects in a stress-induced model of depression, although their underlying mechanisms are slightly different. IMI and RA reversed the stressinduced changes of SUV39H1 and BDNF in the hippocampus. However, unlike RA, IMI did not block GCinduced MKP-1 upregulation in HT22 cells and stress-induced MKP-1 upregulation in the hippocampus (Figs. 4 and 6). FLX reversed the stress-induced changes in SUV39H1, Mkp-1, and BDNF expression in the hippocampus. However, FLX did not reverse the GC-induced Mkp-1 upregulation in HT22 cells (Fig. 4b). FLX and IMI are SSRI and TCA anti-depressants, respectively, and their primary mode of action is to increase monoamine action in synaptic sites. As demonstrated in the present study, FLX and IMI reversed the GC-induced changes of SUV39H1 and BDNF in HT22 cells (Fig. 4a,c). Further studies are needed to establish how FLX and IMI reverse GC-induced changes of SUV39H1, CREB, and BDNF, but produced no effect on Mkp-1 expression, and why FLX produces cytotoxicity in neuronal cells. In a CUS-induced model of depression, FLX reversed the increased level of Mkp-1 in the dentate gyrus, partially blocked increased Mkp-1 in CA1, but not in CA3 cell layers [13. IMI or FLX treatment for 14 days in normal mice (2 M) decreased immobility time in the TST and FST. However, IMI and FLX significantly enhanced anxiety [35]. Furthermore, FLX and IMI in normal mice produced severe bowel problems including swelling of the small intestine, being full with feces and reduced defecation boli [35]. Thus, although FLX and IMI are currently used as antidepressants, animal studies suggest that FLX and IMI appear to exert multiple actions.

As demonstrated in the present study, RA blocked the GC-induced changes of SUV39H1, Mkp-1, and Bdnf expression and overall RA was more effective than IMI and FLX in remediating stress-induced changes of SUV39H1 and Mkp-1 expression (Figs. 4 and 6). Furthermore, RA treatment rescued stress-induced dendritic morphology changes and restored decreased neurogenesis in the hippocampus (Fig. 5). In a chronic stress model of depression, RA increased ERK activity and BDNF expression in the hippocampus, which protects against stress-induced depression-like behaviors [36]. RA is known as a phenolic carboxylic acid that produces antioxidant and anti-inflammatory effects in various cell types [37, 38]. The results of the present study and those of our previous one [22] suggest that RA increases SUV39H1 independently from its antioxidant property or anti-inflammatory effects. RA upregulated SUV39H1 expression and increased SUV39H1 binding to the proximal promoter of the Mkp-1, and thereby reduced Mkp-1 expression [22]. In aged mice, the RA-dependent decrease of Mkp-1 expression is likely to be induced via a SUV39H1-dependent mechanism (Figs. 1 and 2). These results suggest that RA produces antidepressant-like effects by modulating SUV39H1 and Mkp-1-regulated signaling pathways in aging and stress-induced depressive behaviors.

\section{Abbreviations}

Bdnf Brain derived neurotrophic factor

CREB CAMP response element binding protein 
ChIP Chromatin immunoprecipitation

DCX Doublecortin

FLX Fluoxetine

GC Glucocorticoid

IMI Imipramine

MAP2 Microtubule-associated protein 2

Mkp-1 Mitogen-activated protein kinase phosphatase-1

RA Rosmarinic acid

SUV39H1 Suppressor of variegation3-9 homolog 1

\section{Declarations}

\section{Funding and Acknowledgements}

This research was supported by a grant (2021R1A2B5B02002245) from the Ministry of Science, ICT and Future Planning, Republic of Korea.

\section{Conflicts of interest/Competing interests}

The authors declare no competing financial interests.

\section{Code availability}

Not applicable

\section{Authors' contributions}

JEL and SYP carried out the experiments; JEL and PLH designed the experiments, performed the statistical analysis, and wrote the manuscript.

\section{Consent for publication}

All authors consent to the publication of the manuscript in Mol Neurobiol, should the article be accepted by the Editor-in-chief.

\section{Ethics approval and consent to participate}

All animals were handled in accordance with the animal care guidelines of Ewha Womans University (IACUC 16-018). 
Availability of Data and Materials

Data and materials will be made available on reasonable request.

\section{References}

1. Smith, S.M. \& Vale, W.W. The role of the hypothalamic-pituitary adrenal axis in neuroendocrine responses to stress. Dialogues Clin Neurosci. 8, 383-395 (2006).

2. Ulrich-Lai, Y.M. \& Herman, J.P. Neural regulation of endocrine and autonomic stress responses. Nat Rev Neurosci. 10, 397-409 (2009).

3. de Kloet, E.R. From receptor balance to rational glucocorticoid therapy. Endocrinology 155, 2754-69 (2014).

4. Tasker, J.G. \& Herman, J.P. Mechanisms of rapid glucocorticoid feedback inhibition of the hypothalamic-pituitary-adrenal axis. Stress. 14, 398-406 (2011).

5. Myers, B. et al., Glucocorticoid actions on synapses, circuits, and behavior: implications for the energetics of stress. Front Neuroendocrinol. 35, 180-196 (2014).

6. McEwen, B.S., Nasca, C. \& Gray, J.D. Stress effects on neuronal structure: Hippocampus, Amygdala, and Prefrontal Cortex. Neuropsychopharmacology. 41, 3-23 (2016).

7. Radley, J., Morilak, D, Viau V. \& Campeau, S. Chronic stress and brain plasticity: Mechanisms underlying adaptive and maladaptive changes and implications for stress-related CNS disorders. Neurosci Biobehav Rev. 58,79-91 (2016).

8. Lupien, S.J., Leon, M, Santi, S., Convit, A., Tarshish, C., Nair, N.P., Thakur, M., McEwen, B.S., Hauger, R.L. \& Meaney, M.J. Cortisol levels during human aging predict hippocampal atrophy and memory deficits. Nat Neurosci., 1, 69-73 (1998).

9. Mattson, M.P. \& Arumugam, T.V. Hallmarks of brain aging: adaptive and pathological modification by metabolic states. Cell Metab. 27, 1176-1199 (2018).

10. Wilkinson, C.W., Peskind, E.R. \& Raskind, M.A. Decreased Hypothalamic-pituitary adrenal axis sensitivity to cortisol feedback inhibition in human aging. Neuroendocrinology. 65, 79-90 (1997).

11. Johar, H., Emeny, R.T., Bidlingmaier, M., Reincke, M., Thorand, B., Peters, A., Heier, M. \& Ladwig, K. Bluned diurnal cortisol pattern is associated with frailty: a cross-sectional study of 745 participants aged 65 to 90 years. J Clin Endocrinol Metab. 99, E464-468 (2014).

12. Aguilera, G. HPA axis responsiveness to stress: Implications for healthy aging. Exp Gerontol. 46, 9095 (2011).

13. Duric, V., Nanasr, M., Licznerski, P., Schmidt, H.D., Stockmeier, C.A., Simen, A.A., Newton, S.S. \& Duman, R.S. negative regulator of MAP kinase causes depressive behavior. Nat Med. 16, 1328-1332 (2010).

14. Barthas, F., Humo, M., Gilsbach, R., Waltisperger, E., Karatas, M., Leman, S., Hein, L., Belzung, C., Boutillier, A.L., Barrot, M. \& Yalcin, I. Cingulate overexpression of Mitogen-activated protein kinase 
phosphatase-1 as a key factor for depression. Biol Psychiatry. 82, 370-379 (2017).

15. Kondoh, K. \& Nishida, E. Regulation of MAP kinases by MAP kinase phosphatases. Biochimn Biophys Acta. 1773, 1227-1237 (2007).

16. Duman, R.S., Malberg, J., Nakagawa, S. \& D'Sa, C. Neuronal plasticity and survival in mood disorders. Biol Psychiatry. 48, 732-739 (2000).

17. Nair \& Vaidya, Cyclic AMPK response element binding protein and brain-derived neurotrophic factor: molecules that modulate our mood? J Biosci. 31, 423-434 (2006).

18. Foster, T.C., Sharrow, K.M., Masse, J.R., Norris, C.M. \& Kumar, A. Calcineurin links $\mathrm{Ca}^{2+}$ dysregulation with brain aging. $J$ Neurosci. 21, 4066-4073 (2001).

19. von Bohlen und Halbach. Involvement of BDNF in age-dependent alterations in the hippocampus. Front Aging Neuosci. 2, pii:36 (2010).

20. Palomer, E., Martin-Segura, A., Baliyan, S., Ahmed, T., Balschun, E., Venero, C., Martin, M.G. \& Dotti, C.G. Aging triggers a repressive chromatin state at Bdnf promoters in hippocampal neurons. Cell Rep. 16, 2889-2900 (2016).

21. Shipp, L.E., Lee, J.V., Yu, C.Y., Pufall, M., Zhang, P., Scott, D.K. \& Wang, J.C. Transcriptional regulation of human dual specificity protein phosphatase 1 (DUSP1) gene by glucocorticoids. PLoS one, $\mathbf{5}$, e13754 (2010).

22. Lee, J.E., Kwon, H.J., Choi, J. \& Han, P.L. Stress-induced epigenetic changes in hippocampal Mkp-1 promote persistent depressive behaviors. Mol Neurobiol. 56, 8537-8556 (2019).

23. Lee, J.E., Kwon, H.J., Choi, J., Seo, J.S. \& Han, P.L. Aging increases vulnerability to stress-induced depression via upregulation of NADPH oxidase in mice. Commun Biol. 3, 292 (2020).

24. Vialou, V., Feng, J., Robison, A. \& Nestler, E.J. Epigenetic mechanisms of depression and antidepressant action. Annu Rev Pharmacol Toxicol. 53, 59-87 (2013).

25. Klengel, T. \& Binder, E.B. Epigenetics of stress-related psychiatric disorders and gene $x$ environment interactions. Neuron. 86, 1343-1357 (2015).

26. Park, J.Y., Kim, T.K., Choi, J., Lee, J.E., Kim, H., Lee, E.H. \& Han, P.L. Implementation of a Twodimensional behavior matrix to distinguish individuals with differential depression states in a rodent model of depression. Exp Neurobiol. 23, 215-223 (2014).

27. Kim, T.K., Kim, J.E., Choi, J., Park, J.Y., Lee, J.E., Lee, Y., Kim, B.Y., Oh, Y,J. \& Han, P.L. Local interleukin18 system in the basolateral amygdala regulates susceptibility to chronic stress. Mol Neurobiol. 54, 5347-5358 (2017).

28. Seo, J.S., Park, J.Y., Choi, J., Kim, T.K., Shin, J.H., Lee, J.K. \& Han, P.L. NADPH oxidase mediates depressive behavior induced by chronic stress in mice. J Neurosci. 32, 9690-9699 (2012).

29. Kim, T.K. \& Han, P.L. Functional connectivity of basolateral amygdala neurons carrying orexin receptors and melanin-concentrating hormone receptors in regulating sociability and mood-related behaviors. Exp Neurobiol. 25, 307-317 (2016). 
30. Kim, T.K., Lee, J.E., Kim, J.E., Park, J.Y., Choi, J., Kim, H., Lee, E.H. \& Han, P.L. G9a-mediated regulation of OXT and AVP expression in the basolateral amygdala mediates stress-induced lasting behavioral depression and its reversal by exercise. Mol Neurobiol. 53, 2843-2856 (2016).

31. Qi, X., Lin, W., Li, J., Li, H., Wang, W., Wang, D. \& Sun, M. Fluoxetine increases the activity of the ERKCREB signal system and alleviates the depressive-like behavior in rats exposed to chronic forced swim stress. Neurobiol Dis. 31, 278-285 (2008).

32. Duman, R.S. \& Voleti, B. Signaling pathways underlying the pathophysiology and treatment of depression: novel mechanisms for rapid-acting agents. Trends Neurosci. 35, 47-56 (2012).

33. Yoo, J., Lee, B.D., Sok, D., Ma, J.Y. \& Kim, M.R. Neuroprotective action of N-acetyl serotonin in oxidative stress-induced apoptosis through the activation of both TrkB/CREB/BDNF pathway and Akt/Nrf2/Antioxidant enzyme in neuronal cells. Redox Biol. 11, 592-599 (2017).

34. Jin, Y., Sun, L.H., Yang, W., Cui, R.J. \& Xu, S.B. The role of BDNF in the neuroimmune axis regulation of mood disorders. Front Neurol. 10, 515 (2019).

35. Baek, I.S., Park, J.Y. \& Han, P.L. Chronic antidepressant treatment in normal mice induces anxiety and impairs stress-coping ability. Exp Neurobiol. 24, 156-168 (2015).

36. Jin, X., Liu, P., Yang, F., Zhang, Y. \& Miao, D. Rosmarinic acid ameliorates depressive-like behaviors in a rat model of CUS and Up-regulates BDNF levels in the hippocampus and hippocampal-derived astrocytes. Neurochem Res. 38, 1828-1837 (2013).

37. Gharrari, H., Venkataramana, M., Ghassam, B.J., Nayaka, S.C., Nataraju, A., Geetha, N.P. \& Prakash, H.S. Rosmarinic acid mediated neuroprotective effects against $\mathrm{H} 2 \mathrm{O} 2$-induced neuronal cell damage in N2A cells. Life Sci. 113, 7-13 (2014).

38. Andrade, J.M., Faustino, C., Garcia, C., Ladeiras, D., Reis, C.P. \& Rijo, P. Rosmarinus officinalis L.: an update review of its phytochemistry and biological activity. Future Sci OA. 4, FSO283 (2019).

\section{Figures}


a

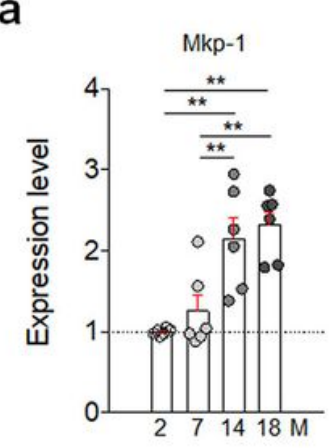

b

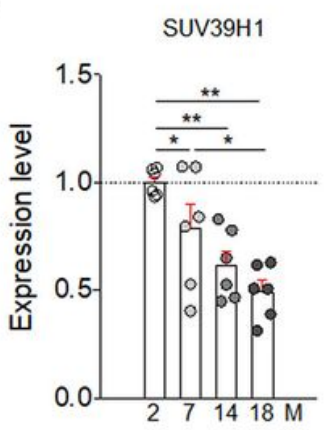

C
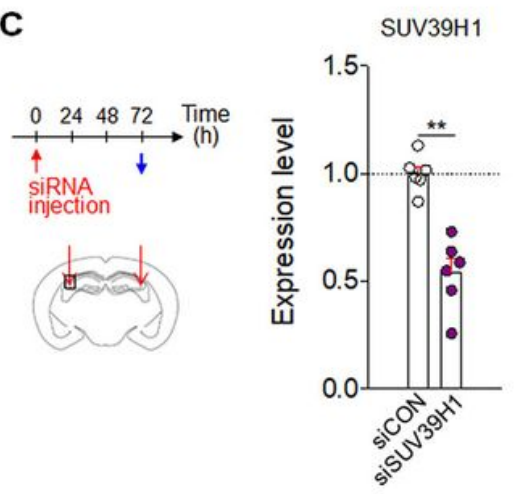

g

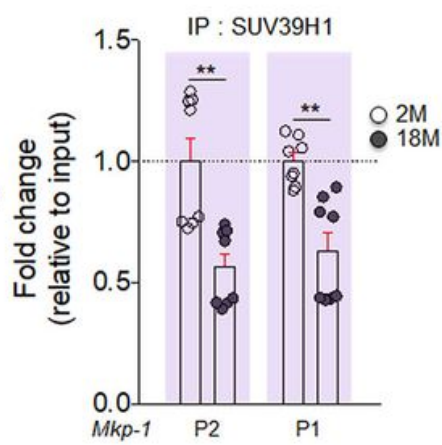

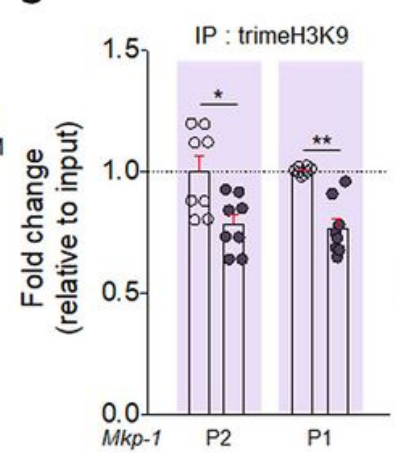

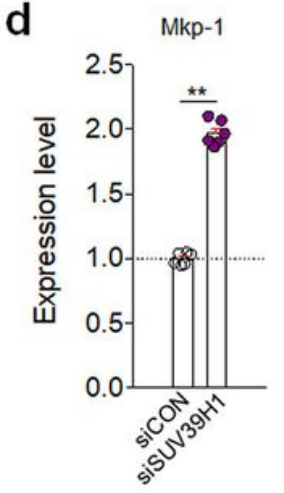

h

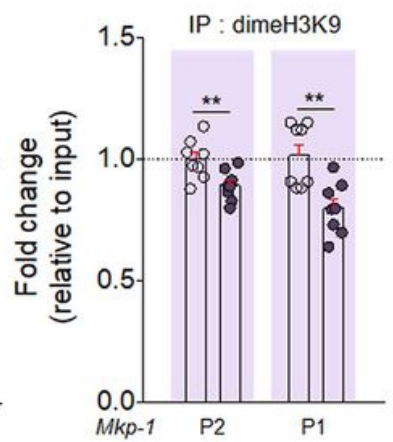

\section{Figure 1}

Aging upregulated Mkp-1 expression via SUV39H1 downregulation in the hippocampus. a,b Transcript levels of Mkp-1 (a) and SUV39H1 (b) in the hippocampus of mice at 2, 7, 14, and 18 months of age $(n=6$, each; One-way ANOVA, $F(3,20)=12.72, p<0.0001$ for Mkp-1; $n=6$, each; One-way ANOVA, $F(3,20)=$ 9.562, $p=0.0004$ for SUV39H1). c,d Experimental design (c, left panel) for stereotaxic injection of siRNASUV39H1 and siRNA-CON in CA3 region (red arrows). Blue arrow, tissue preparation point. Transcript levels of and SUV39H1 (c, right panel) and Mkp-1 (d) after siRNA-mediated knockdown of SUV39H1 in the CA3 region ( $n=6$, each). e-h Diagrams (e) showing the promoter region of the Mkp-1 and the process of immunoprecipitation, DNA purification, and following $\mathrm{QPCR}$ analysis. GRE, glucocorticoid-responsive element (red box). P1 and P2, the promoter regions used for ChIP-qPCR analysis. ChIP-qPCR data showing the levels of SUV39H1 (f), trimeH3K9 (g), and dimeH3K9 (h) binding to the promoter of the Mkp1 in the hippocampus of mice at 2 and 18 months of age $(n=8$, each). Data are presented as mean \pm SEM. * and **, differences between indicated groups at $p<0.05$ and $p<0.01$, respectively (Student's t-test, and one-way ANOVA followed by Newman-Keuls post hoc test). 

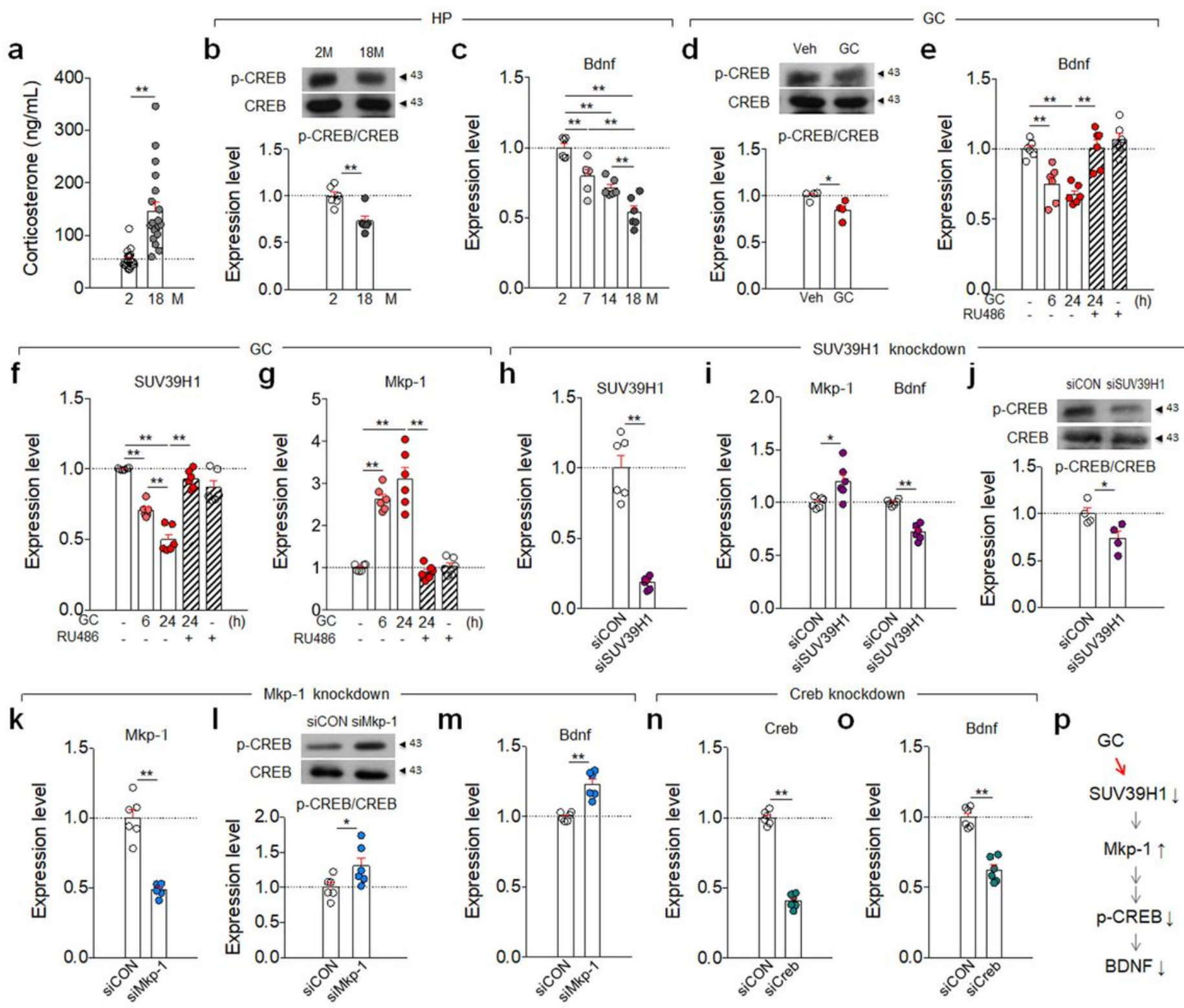

\section{Figure 2}

Glucocorticoid or aging-induced changes in the SUV39H1-Mkp-1-p-CREB-Bdnf pathway. a Serum corticosterone levels of mice at 2 months and 18 months of age $(n=20$, each). b Western blot data showing p-CREB and CREB levels in the hippocampus of mice at 2 months and 18 months $(n=6$, each). $c$ Transcript levels of Bdnf in the hippocampus of mice at 2, 7, 14, and 18 months of age ( $n=6$, each; Oneway ANOVA, $F(3,20)=25.62, p<0.0001)$. $d$ Western blot data showing $p$-CREB and CREB levels in HT22 cells treated with GC (400 ng/ml) for $24 \mathrm{~h}(\mathrm{n}=4$, each). e-g Transcript levels of Bdnf (e), SUV39H1 (f), and Mkp-1 (g) in HT22 cells treated with GC (400 ng/ml) for $6 \mathrm{~h}$ or $24 \mathrm{~h}$, or GC (24 h) plus RU486, and RU486 alone. Bdnf ( $n=6$, each; CON vs. GC6h vs. GC24h, One-way ANOVA, F(2,15) = 20.34, $p<0.0001$; CON vs. GC24h vs. CON+RU486 vs. GCh+RU486, Two-way ANOVA, GC main effects, $F(1,20)=22.27, p<0.0001$; RU486 main effects, $F(1,20)=23.72, p<0.0001 ; G C \times R U 486, F(1,20)=10.28, p=0.0044)$. SUV39H1 ( $n=$ 6 , each; CON vs. GC6h vs. GC24h, One-way ANOVA, F(2,15) = 102.6, $p<0.0001 ;$ CON vs. GC24h vs. 
CON+RU486 vs. GCh+RU486, Two-way ANOVA, GC main effects, $F(1,20)=21.45, p=0.0002 ; R U 486$ main effects, $F(1,20)=46.71, p<0.0001 ; G C \times R U 486, F(1,20)=76.23, p<0.0001)$. Mkp-1 ( $n=6$, each; CON vs. GC6h vs. GC24h, One-way ANOVA, $F(2,15)=40.21, p<0.0001$; CON vs. GC24h vs. CON+RU486 vs. GCh+RU486, Two-way ANOVA, GC main effects, $F(1,20)=43.29, p<0.0001 ; R U 486$ main effects, $F(1,20)$ $=52.94, p<0.0001 ; G C \times$ RU486, $F(1,20)=56.14, p<0.0001)$. $h$ Transcript levels of SUV39H1 in HT22 cells treated with siRNA-SUV39H1 or siRNA-CON $(H)(n=6$, each). i Transcript levels of Mkp-1 and Bdnf after SUV39H1 knockdown in HT22 cells. $(n=6$, each). j Western blot data showing p-CREB and CREB levels after SUV39H1 knockdown in HT22 cells ( $n=4$, each). $k$ Transcript levels of Mkp-1 in the HT22 cells treated with siRNA-Mkp-1 or siRNA-CON. $(n=6$, each). I Western blot data showing p-CREB and CREB levels after Mkp-1 knockdown in HT22 cells $(n=4$, each). $m$ Transcript levels of Bdnf after Mkp-1 knockdown in HT22 cells ( $n=6$, each). $n$ Transcript levels of Creb in the HT22 cells treated with siRNACreb or siRNA-CON ( $n=6$, each). o Transcript levels of Bdnf changed by Creb knockdown in HT22 cells ( $n$ $=6$, each). $\mathrm{p} \mathrm{A}$ summary of the SUV39H1, Mkp-1, p-CREB, and BDNF signaling pathway. Data are presented as mean \pm SEM. * and ${ }^{*}$, differences between indicated groups at $p<0.05$ and $p<0.01$, respectively (Student's t-test, and one-way ANOVA or two-way ANOVA followed by Newman-Keuls post hoc test). 
a

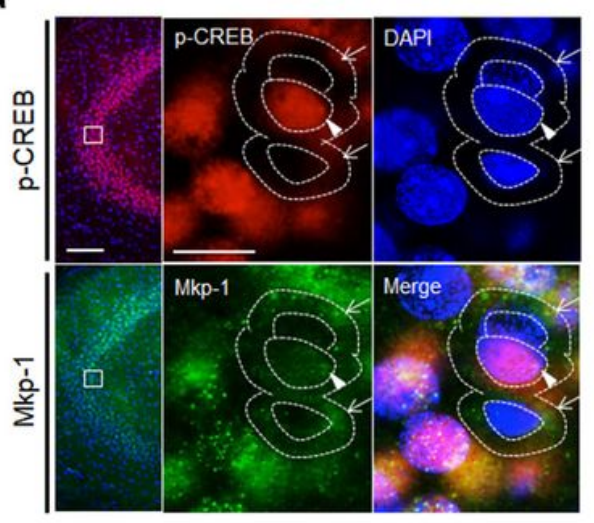

c

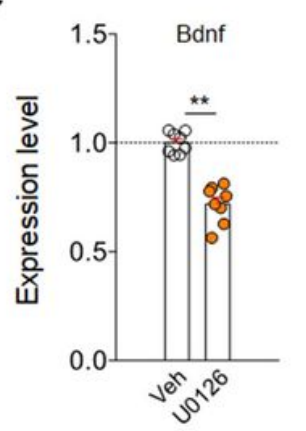

f

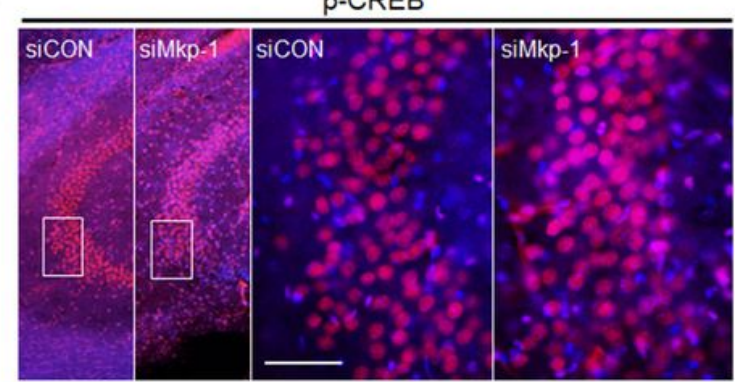

b

d
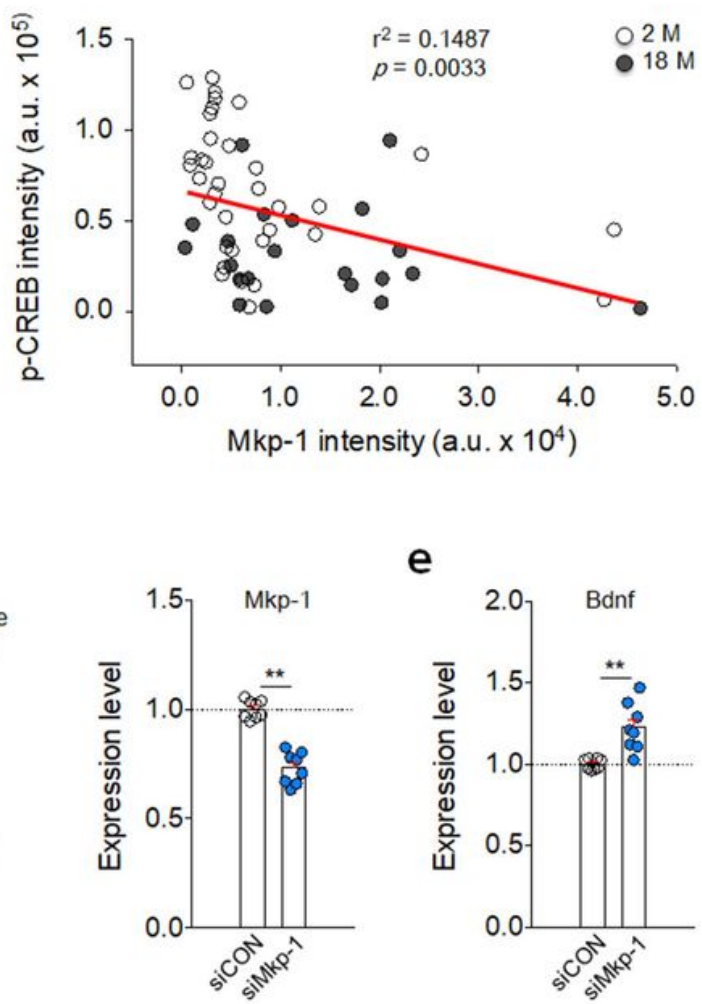

e

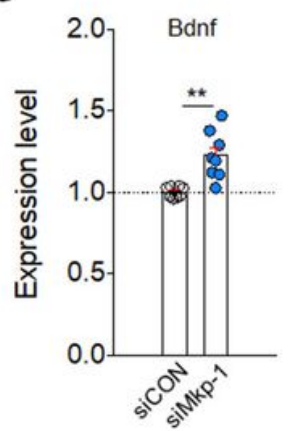

g

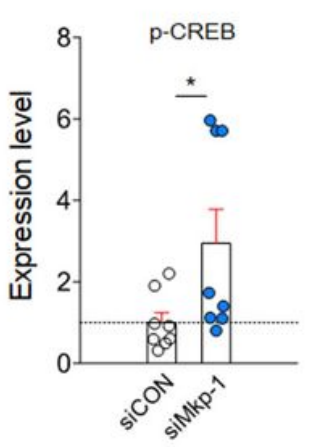

h $G C$ SUV39H1 Mkp-1 $\uparrow$ p-ERK $\downarrow$ p-CREB $\downarrow$ BDNF

\section{Figure 3}

Mkp-1 negatively regulated Bdnf expression via downregulation of $p$-CREB. $a, b$ Photomicrographs showing Mkp-1 and p-CREB expression in CA3 pyramidal neurons of young (2 M) and aged (18 M) mice (a). Scale bars, $100 \mu \mathrm{m}$ (left); $20 \mu \mathrm{m}$ (right). Quantification levels of p-CREB and Mkp-1 expression in individual neurons $(b)$. ( $n=35$ cells for $2 M, n=21$ cells for $18 M)$. c Transcript levels of Bdnf in the HT22 cells treated with U0126 (10 $\mu \mathrm{M})(\mathrm{n}=8$, each). d,e. Experimental design (d, left panel) for stereotaxic injection of siRNA-Mkp-1 and siRNA-CON in CA3 regions (red arrows). Blue arrow, tissue preparation point. Transcript levels of and Mkp-1 (d, right panel) and Bdnf (e) after Mkp-1 knockdown in CA3 regions of mice ( $n=8$, each). $f, g$ Photomicrographs showing p-CREB levels in pyramidal neurons of the CA3 regions infused with siRNA-Mkp-1 and siRNA-CON (f). Scale bars, $100 \mu \mathrm{m}$ (left); $20 \mu \mathrm{m}$ (right). Quantification levels of $\mathrm{p}$-CREB expression in CA3 pyramidal neurons $(\mathrm{g})$. $(\mathrm{n}=8$, each). h A summary of the signaling pathway of SUV39H1, Mkp-1, p-ERK, p-CREB, and BDNF. Data are presented as mean \pm 
SEM. * and **, differences between indicated groups at $p<0.05$ and $p<0.01$, respectively (Student's t-test, and one-way ANOVA followed by Newman-Keuls post hoc test).

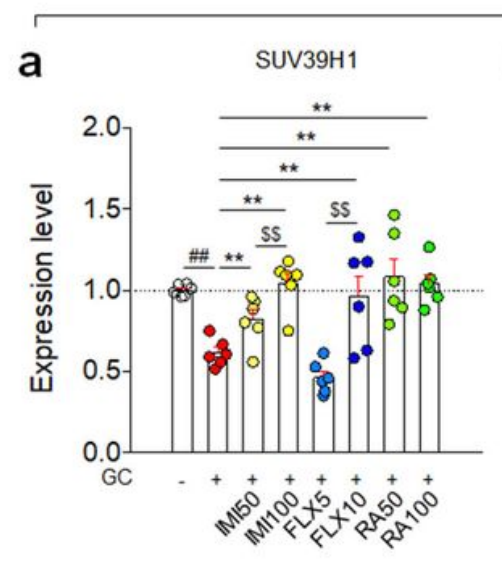

e

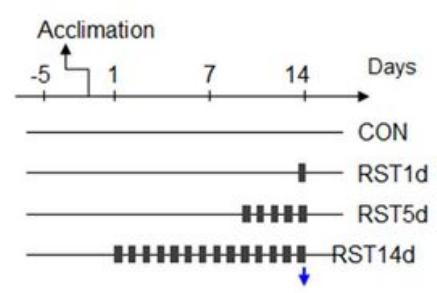

i
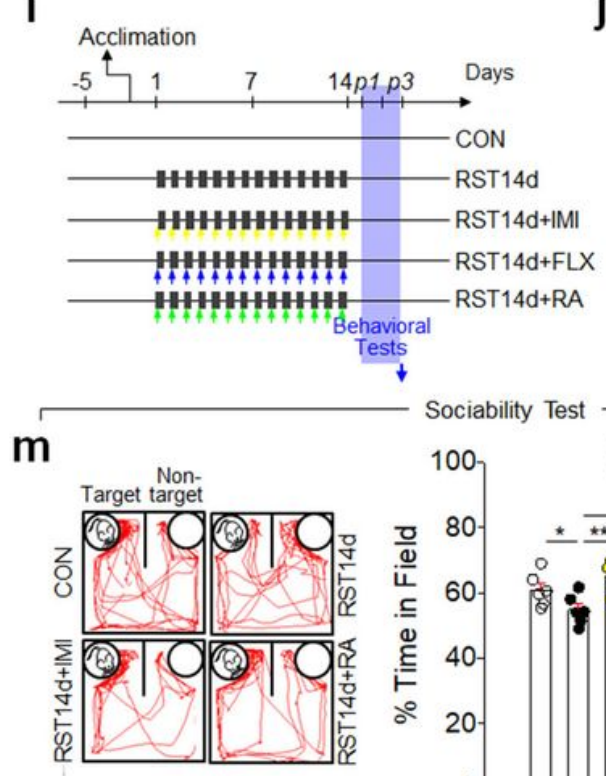

b

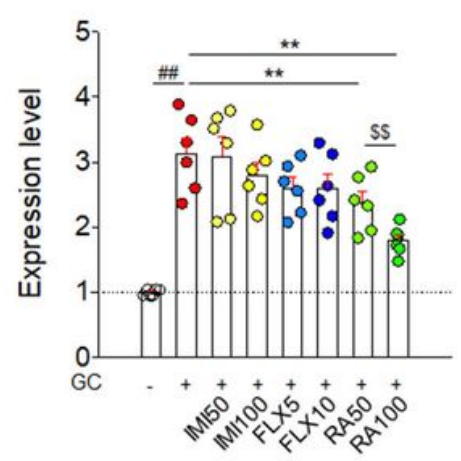

f

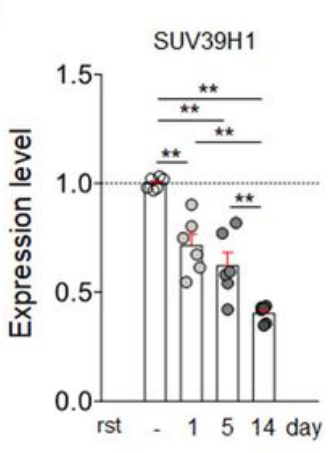

j 2.0 suv39H1 k

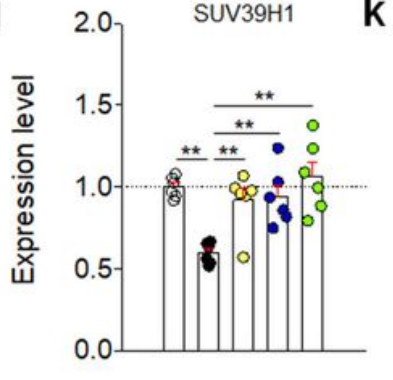

C

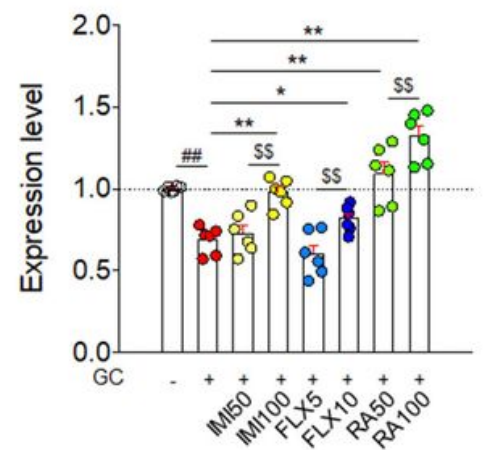

g

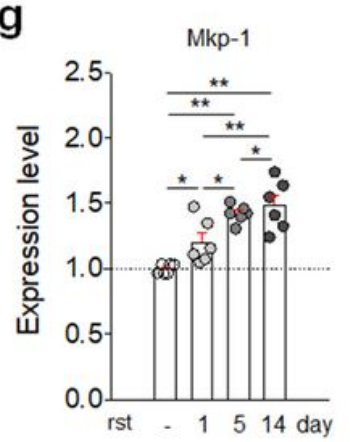

h
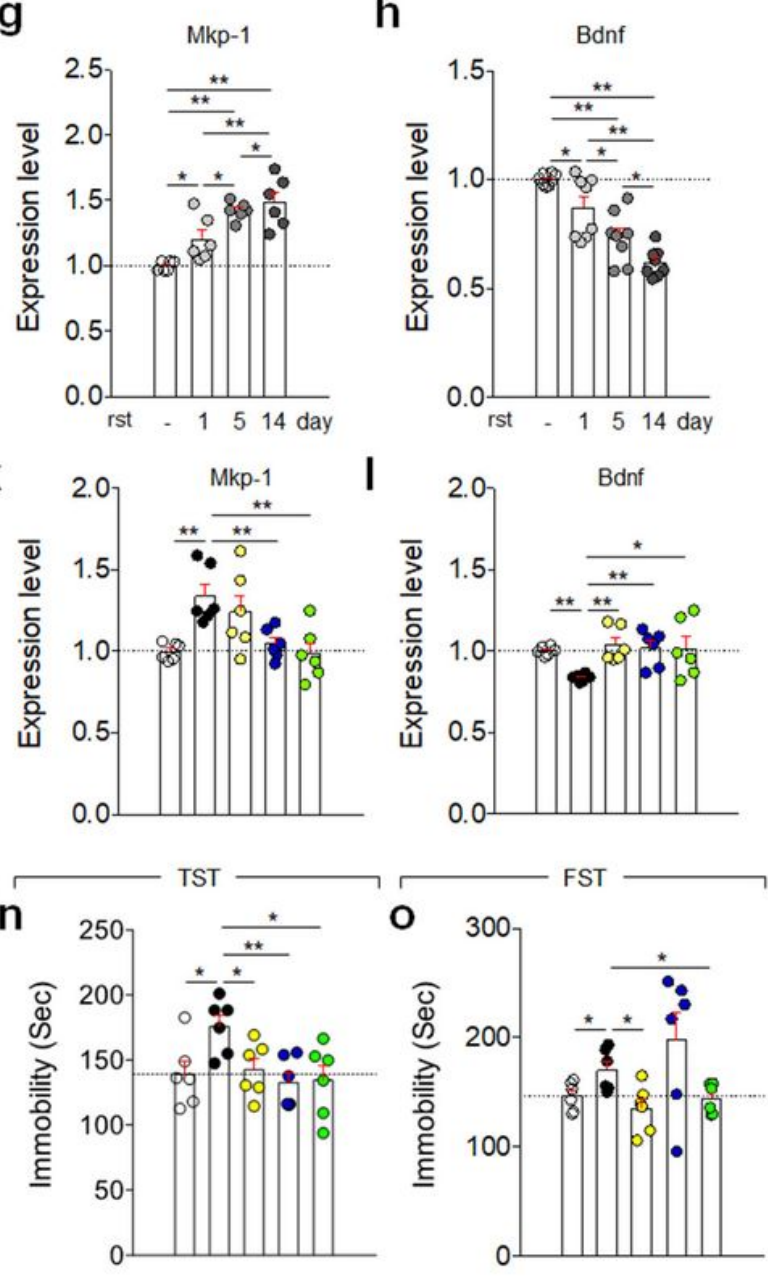

d

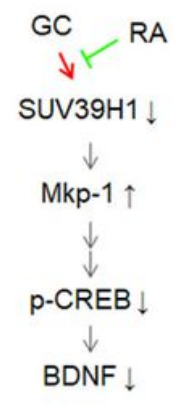

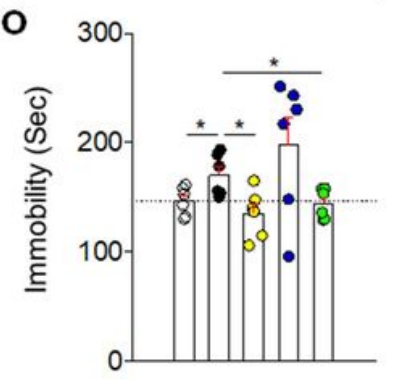

\section{Figure 4}

RA blocked GC- or stress-induced changes of the SUV39H1-Mkp-1-Bdnf pathway. a-c Transcript levels of SUV39H1 (a), Mkp-1 (b), and Bdnf (c) in the HT22 cells treated with GC (400 ng/ml), GC plus IMI (50 or $100 \mu \mathrm{M}), \mathrm{GC}$ plus FLX (5 or $10 \mu \mathrm{M})$ or GC plus RA (50 or $100 \mu \mathrm{M})(\mathrm{n}=6$, each; SUV39H1, One-way ANOVA, for IMI, $F(3,20)=17.58, p<0.0001$; for $F L X, F(3,20)=14.75, p<0.0001$; for $R A, F(3,20)=11.47, p=$ 
0.0001; Mkp-1, One-way ANOVA, for IMI, $F(3,20)=20.59$, $p<0.0001$; for $F L X, F(3,20)=25.63, p<0.0001$; for RA, $F(3,20)=33.26, p<0.0001$; Bdnf, One-way ANOVA, for IMI, $F(3,20)=21.64$, $p<0.0001$; for $F L X$, $F(3,20)=22.5, p<0.0001$; for RA, $F(3,20)=27.31, p<0.0001)$. $d$ A summary of the SUV39H1, Mkp-1, $p-$ CREB, and BDNF signaling pathway. e Experimental design for treatment with daily 2 h-restraint for 1 day, 5 days, or 14 days. Arrow, time point for tissue preparation. f-h Transcript levels of SUV39H1 (f), Mkp-1 $(\mathrm{g})$, and $\mathrm{Bdnf}(\mathrm{h})$ in the hippocampus of mice treated with 2-h restraint for the number of days indicated $(n=6$ for SUV39H1 and Mkp-1, $n=8$ for Bdnf; SUV39H1, One-way ANOVA, $F(3,20)=35.68, p<0.0001$; Mkp-1, One-way ANOVA, $F(3,20)=16.33, p<0.0001$; Bdnf, One-way ANOVA, $F(3,28)=22.8, p<0.0001)$. i Experimental design for treatment with daily 2-h restraint for 14 days (RST14d) or RST14d plus IMI (20 $\mathrm{mg} / \mathrm{Kg}$ ), RST14d plus FLX (20 mg/Kg), or RST14d plus RA (30 mg/Kg), and following behavioral tests. Arrow, time point for tissue preparation. $\mathrm{j}-\mathrm{I}$ Transcript levels of SUV39H1 (j), Mkp-1 (k), and Bdnf (I) in the hippocampus of mice treated RST14d, RST14d plus IMI, RST14d plus FLX, or RST14d plus RA ( $n=6$, each; SUV39H1, One-way ANOVA, for IMI, $F(2,15)=21.01, p<0.0001$; for $F L X, F(2,15)=21.74, p<0.0001$; for RA, $F(2,15)=20.69, p<0.0001$; Mkp-1, One-way ANOVA, for IMI, $F(2,15)=5.848, p=0.0133$; for $F L X$, $F(2,15)=14.08, p=0.0004$; for RA, $F(2,15)=11.98, p=0.0008$; Bdnf, One-way ANOVA, for IMI, $F(2,15)=$ $16.57, p=0.0002$; for FLX, $F(2,15)=13.79, p=0.0004$; for $R A, F(2,15)=5.51, p=0.0161) . m$

Representative tracking plots (left panels) and time spent in the target and non-target fields in the sociability test of mice treated RST14d, RST14d plus IMI, RST14d plus FLX, or RST14d plus RA. $(n=6$ mice/group; target field, One-way ANOVA, for IMI, $F(2,15)=6.488, p=0.0093$; for $F L X, F(2,15)=2.377, p=$ 0.1268; for RA, $F(2,15)=5.119, p=0202$; non-target field, One-way ANOVA, for IMI, $F(2,15)=6.49, p=$ 0.0093 ; for $F L X, F(2,15)=2.375, p=0.127$; for $R A, F(2,15)=5.12, p=0202)$. n,o Immobility time in the TST and FST of mice treated RST14d, RST14d plus IMI, RST14d plus FLX, or RST14d plus RA ( $n=6$ mice/group; TST, One-way ANOVA, for IMI, $F(2,15)=4.935, p=0.0225$; for $F L X, F(2,15)=6.826, p=$ 0.0078; for RA, $F(2,15)=5.022, p=0.0214$; FST, One-way ANOVA, for IMI, $F(2,15)=5.695, p=0.0145$; for $F L X, F(2,15)=2.709, p=0.0990$; for $R A, F(2,15)=5.118, p=0.0202)$. Data are presented as mean $\pm S E M$. * and $* *$, differences between indicated groups at $p<0.05$ and $p<0.01$, respectively (Student's t-test, and one-way ANOVA followed by Newman-Keuls post hoc test). 
a

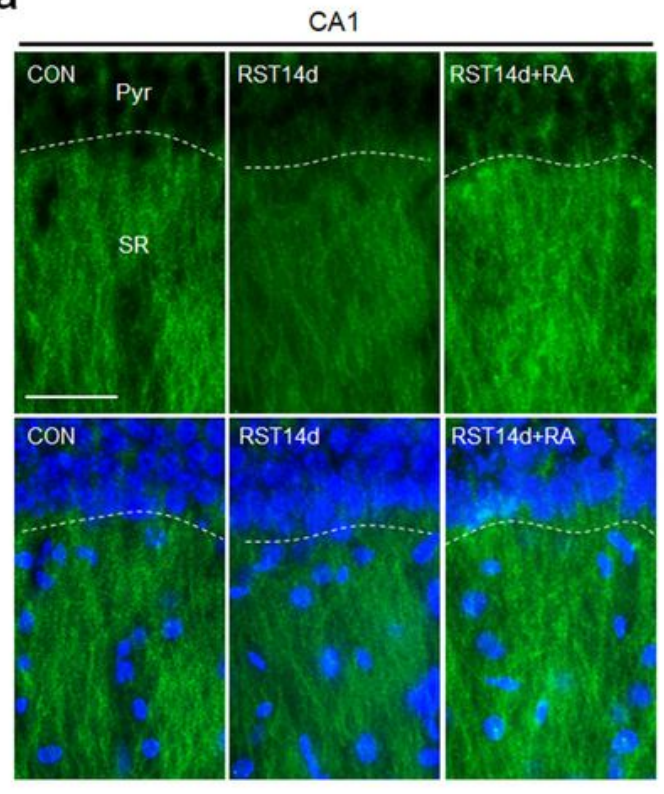

e

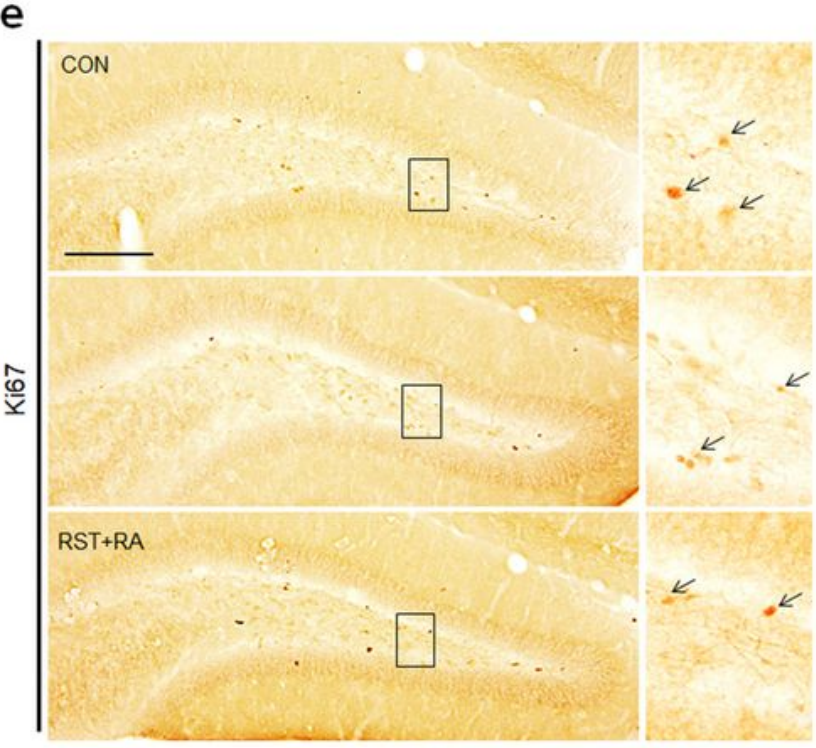

f

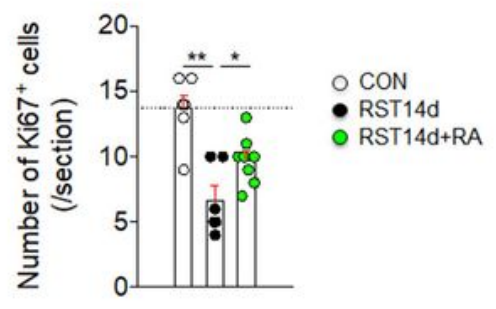

b

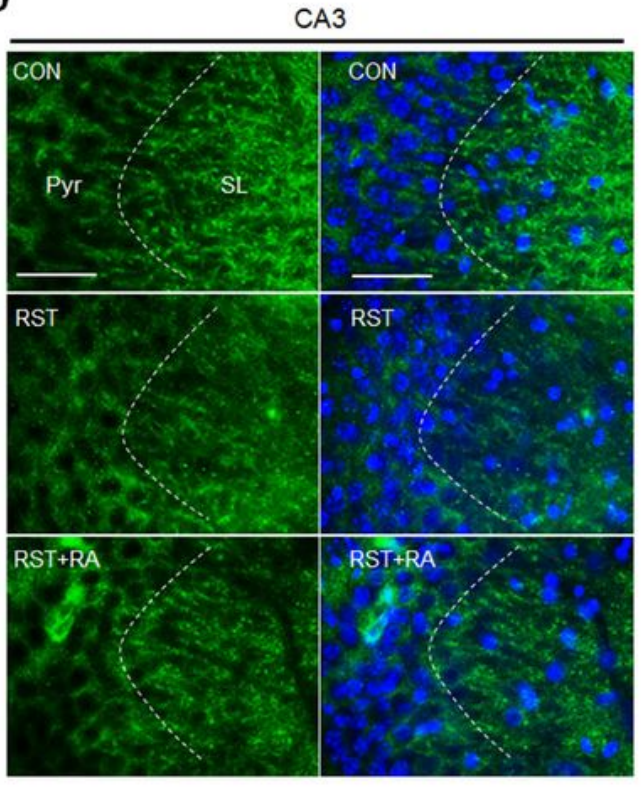

g

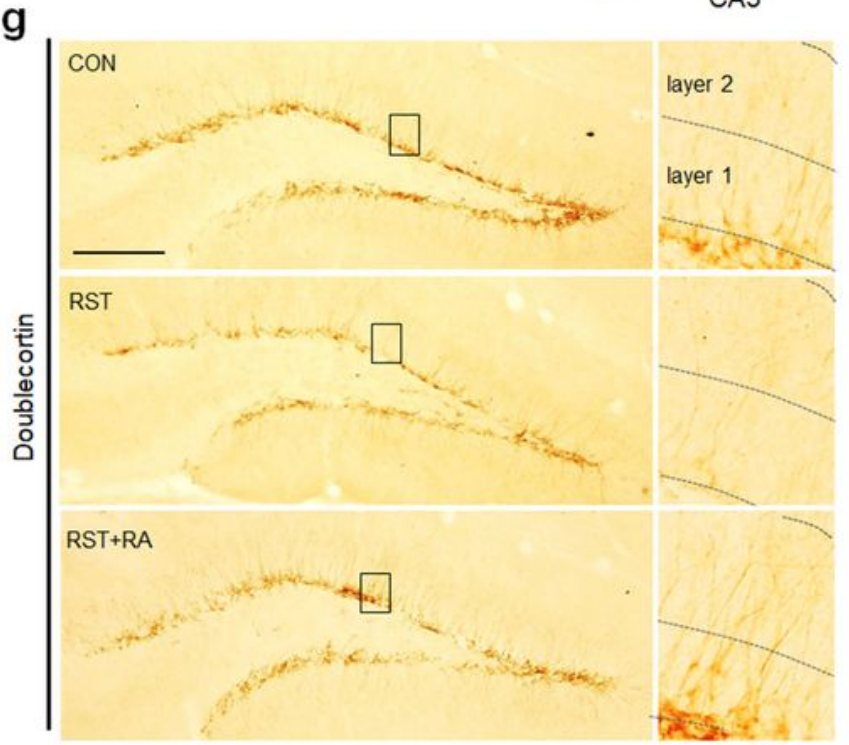

h

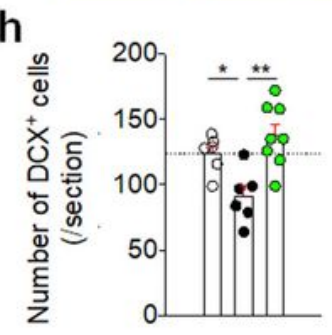

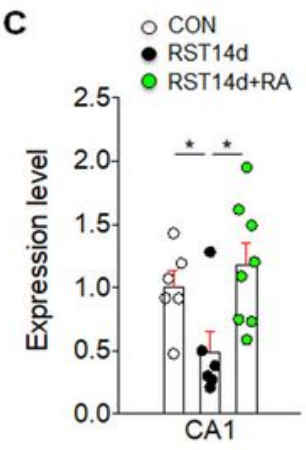

d

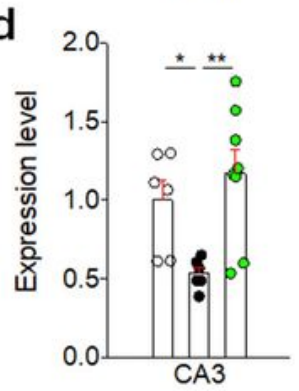


DAPI staining levels, in the CA1 (c) and CA3 (d). ( $n=6$ mice for CON or RST groups, $n=8$ mice for RST+RA; One-way ANOVA, for CA1, $F(2,17)=4.827, p=0.0219$; for CA3, $F(2,17)=6.742, p=0.007)$. e,f Photomicrographs showing the DG stained with anti-Ki67 in mice treated with RST14d or RST14d plus RA (e). Scale bar, $200 \mu \mathrm{m}$. Number of Ki67-positive cells in the DG (f) ( $n=6$ mice for CON and RST, $n=8$ mice for RST+RA; One-way ANOVA, F(2,17) = 13.63, $p=0.003)$. g-i Photomicrographs showing the DG stained with anti-Doublecortin (DCX) in mice treated with RST14d or RST14d plus RA (g). Scale bar, 200 $\mu \mathrm{m}$. Number of DCX-positive cells in the DG $(h)(n=6$ mice for CON and RST, $n=8$ mice for RST+RA; Oneway ANOVA, $F(2,17)=9.184, p=0.002)$. Relative intensity of developing dendritic processes stained by anti-DCX in the arbitrary layers 1 and 2 (i) ( $n=6$ mice for CON or RST, $n=8$ mice for RST+RA; One-way ANOVA, for layer $1, F(2,17)=5.991, p=0.0107$; for layer $2, F(2,17)=4.704, p=0.0237)$. Data are presented as mean \pm SEM. * and $* *$, difference between indicated groups at $p<0.05$ and $p<0.01$, respectively (Student's t-test, and one-way ANOVA followed by Newman-Keuls post hoc test).
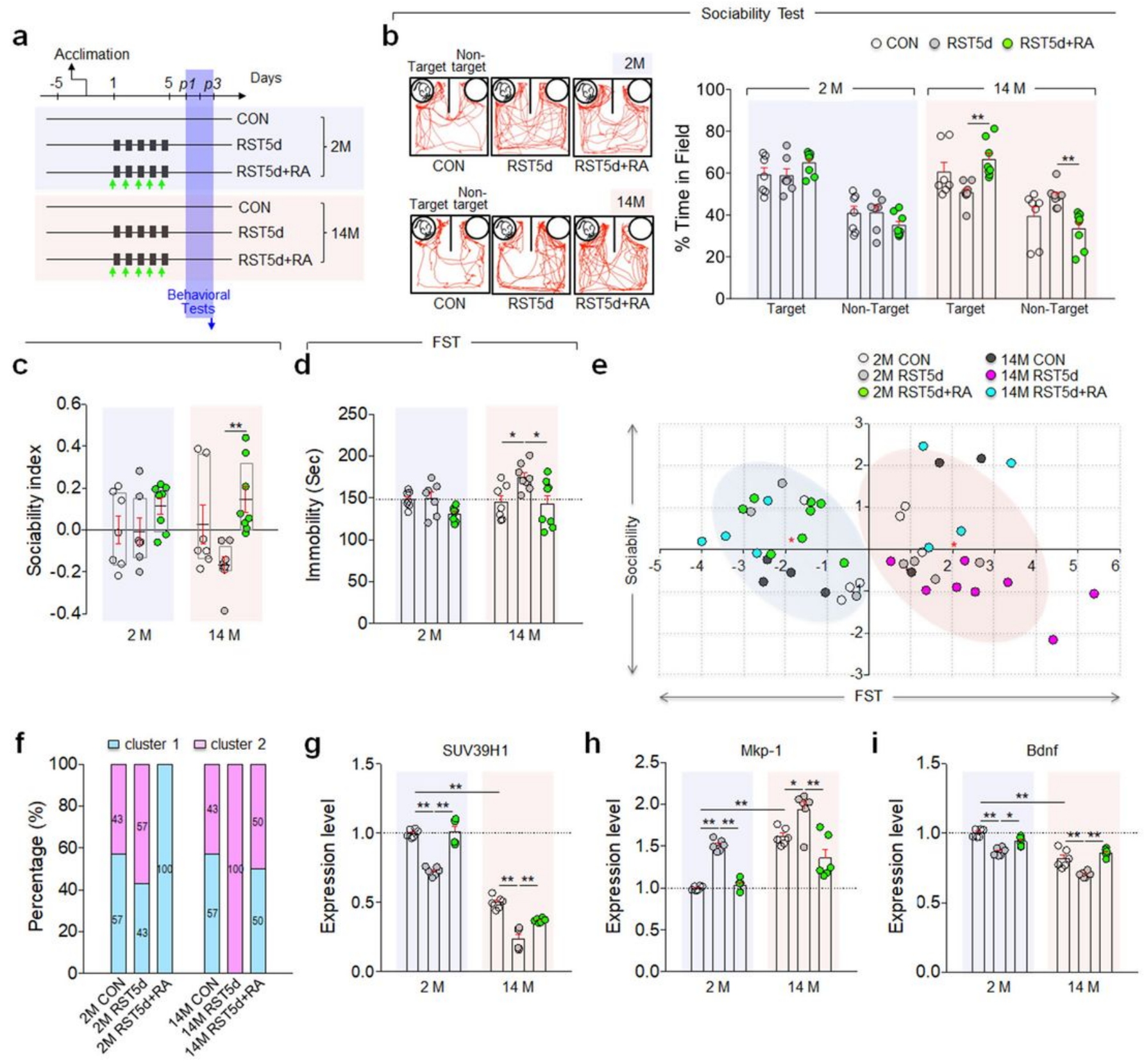


\section{Figure 6}

RA protected against stress-induced changes of the SUV39H1-Mkp-1-Bdnf pathway and depression-like behaviors in aged mice. a Experimental design for treatment with daily 2-h restraint for 5 days (RST5d) or RST5d plus RA (30 mg/Kg) in young (2 M) and aged (14 M) mice, and following behavioral tests. Arrow, time point for tissue preparation. $b$ Representative tracking plots and time spent in the target and nontarget fields in the social interaction test of mice treated RST5d or RST5d plus RA in young and aged mice ( $n=7$ mice/group for $2 M$ CON, 2M RST5d, and 14M CON, $n=8$ mice/group for 2M RST5d+RA, $14 M$ RST5d, and 14M RST5d+RA; target field, Two-way ANOVA, age main effects, $F(1,39)=0.4917, p=0.4873$; RST5d main effects, $F(2,39)=6.569, p=0.0035$; age $x$ RST5d, $F(2,39)=1.583, p=0.2182$; non-target field, Two-way ANOVA, age main effects, $F(1,39)=0.3981, p=0.5318$; RST5d main effects, $F(2,39)=$ $6.612, p=0.0034$; age $x$ RST5d, $F(2,39)=1.59, p=0.2168)$. c Sociability index of mice treated RST5d or RST5d plus RA in young and aged mice ( $n=7$ mice/group for $2 M$ CON, 2M RST5d, and $14 M$ CON, $n=8$ mice/group for 2M RST5d+RA, 14M RST5d, and 14M RST5d+RA; Two-way ANOVA, age main effects, $F(1,39)=0.44, p=0.511 ;$ RST5d main effects, $F(2,39)=6.603, p=0.0034$; age $x$ RST5d, $F(2,39)=1.594, p$ $=0.2161) . d$ Immobility time in the FST of young and aged mouse groups treated RST5d or RST $5 d$ plus RA ( $\mathrm{n}=7$ mice/group for $2 \mathrm{M}$ CON, $2 \mathrm{M}$ RST5d, and $14 \mathrm{M}$ CON, $\mathrm{n}=8$ mice/group for $2 \mathrm{M}$ RST5d+RA, $14 \mathrm{M}$ RST5d, and 14M RST5d+RA; Two-way ANOVA, age main effects, $F(1,39)=4.328, p=0.0441$; RST5d main effects, $F(2,39)=7.421, p=0.0019$; age $x$ RST5d, $F(2,39)=2.237, p=0.1203)$. e, , Distribution of individuals of CON, RST5d or RST5d plus RA groups plotting with z-scores (z, standard deviation) in the sociability test x FST matrix, which are grouped into two clusters (blue and pink ellipses) by K-means clustering or can be classified into four quadrants (open ellipses) (e). Proportion of individuals in the two clusters (f) (Cluster $1: 57.14 \%$ for $2 \mathrm{M}$ CON, $42.86 \%$ for $2 \mathrm{M}$ RST $5 \mathrm{~d}, 100 \%$ for $2 \mathrm{M}$ RST $5 \mathrm{~d}+\mathrm{RA}, 57.14 \%$ for $14 \mathrm{M}$ CON, $0 \%$ for $14 \mathrm{M}$ RST $5 \mathrm{~d}$, and $50 \%$ for $14 \mathrm{M}$ RST5d+RA; Cluster $2: 42.86 \%$ for $2 \mathrm{M} \mathrm{CON}, 57.14 \%$ for $2 \mathrm{M}$ RST $5 \mathrm{~d}, 0 \%$ for $2 \mathrm{M}$ RST+RA, $42.86 \%$ for $14 \mathrm{M}$ CON, $100 \%$ for $14 \mathrm{M}$ RST $5 \mathrm{~d}$, and $50 \%$ for $14 \mathrm{M}$ RST5d+RA). g-i Transcript levels of SUV39H1 (g), Mkp-1 (h), and Bdnf (i) in the hippocampus of young or aged mouse groups treated with RST5d and RST5d plus RA ( $n=6$, each; SUV39H1, two-way ANOVA, age main effects, $F(1,30)=845.4, p<0.0001$; RST5d main effects, $F(2,30)=75.96, p<0.0001$; age $x$ RST5d, $F(2,30)=6.966, p=0.0033 ;$ Mkp-1, two-way ANOVA, age main effects, $F(1,30)=81.88, p<0.0001$, RST5d main effects, $F(2,30)=40.72, p<0.0001$; age $x$ RST5d, $F(2,30)=2.871, p=0.0723$; Bdnf, two-way ANOVA, age main effects, $F(1,30)=130.3, p<0.0001$; RST5d main effects, $F(2,30)=40.64, p<0.0001$; age $x$ RST5d, $F(2,30)=6.28, p=0.0053)$. Data are presented as mean $\pm S E M . *$ and ${ }^{* *}$, differences between indicated groups at $p<0.05$ and $p<0.01$, respectively (Student's t-test, and one-way ANOVA or two-way ANOVA followed by Newman-Keuls post hoc test). 